\title{
The Recoverable Robust Facility Location Problem
}

\author{
Eduardo Álvarez-Miranda ${ }^{\mathrm{a}, 1}$, Elena Fernández ${ }^{\mathrm{b}}$, Ivana Ljubićc \\ ${ }^{a}$ Industrial Engineering Department, Universidad de Talca, Curicó, Chile \\ ${ }^{b}$ Departament of Statistics and Operational Research, Universidad Politécnica de Catalunya, Barcelona, \\ Spain \\ ${ }^{c}$ Department of Statistics and Operations Research, Universität Wien, Vienna, Austria
}

\begin{abstract}
This work deals with a facility location problem in which location and allocation (transportation) policy is defined in two stages such that a first-stage solution should be robust against the possible realizations (scenarios) of the input data that can only be revealed in a second stage. This solution should be robust enough so that it can be recovered promptly and at low cost in the second stage. In contrast to some related modeling approaches from the literature, this new recoverable robust model is more general in terms of the considered data uncertainty; it can address situations in which uncertainty may be present in any of the following four categories: provider-side uncertainty, receiver-side uncertainty, uncertainty in-between, and uncertainty with respect to the cost parameters.

For this novel problem, a sophisticated branch-and-cut framework based on Benders decomposition is designed and complemented by several non-trivial enhancements, including scenario sorting, dual lifting, branching priorities, matheuristics and zero-half cuts. Two large sets of instances that incorporate spatial and demographic information of countries such as Germany and US (transportation) and Bangladesh and the Philippines (disaster management) are introduced. They are used to analyze in detail the characteristics of the proposed model and the obtained solutions as well as the effectiveness, behavior and limitations of the designed algorithm.
\end{abstract}

Keywords: Facility Location, Two-Stage Robust Optimization, Branch-and-Cut, $L$-Shaped Cuts

\section{Introduction}

Nowadays, we are more and more aware of the growing presence of dynamism and uncertainty in decision making. Fortunately, as the decisions become more complex, the availability of modeling, algorithmic and computational tools increases as well. Facility location and allocation decisions are among the most relevant decisions in several private and public transportation contexts and they usually involve strategic and operative policies with mid and long term impacts. Precisely because of the practical relevance of these decisions, it

\footnotetext{
${ }^{1}$ Corresponding author: Industrial Engineering Department, Universidad de Talca, Merced 437, Curicó, Chile (ealvarez@utalca.cl, Tel: +56-075-201706).
} 
is important that they incorporate the uncertainty that naturally appears during the planning, modeling and operative process. Such uncertainty can be represented by different realizations of the input data: customers that actually require a commodity or a service, locations where the facilities can be located, the transportation network that can be used for connecting customers with facilities, and the corresponding costs. The true values of this data usually become available later in the decision process. In such cases a standard deterministic optimization model that considers a single possible outcome of the input data can lead towards solutions that are unlikely to be optimal, or for that matter even feasible, for the final data realization.

Supply chain management is a strategical area in which both uncertainty and facility location are core elements. For instance, as it is pointed out in [45], supply chains are particularly vulnerable to disruptions (intentional or accidental), imposing the need of taking into account the possible availability of depots and roads and different structures of the demand. Likewise, short-term phenomena such as fluctuations in commodity prices (such as oil) or long-term public policies (such as new toll road concessions) might lead to operational cost increases that should be considered when deciding the transportation network to be used.

In another context, natural events such as tsunamis, hurricanes or blizzards can produce disastrous effects with unpredictable intensity on populated areas and on the transportation infrastructure. Countries such as Bangladesh and the Philippines are two typical examples; both of them are regularly hit by hydrological disasters such as floods and typhoons. According to the Department of Disaster Management of Bangladesh [22], every year around $18 \%$ of the country is flooded, which produces over 5000 causalities and the destruction of more than 7 millions of homes. However, flooded areas my exceed the $75 \%$ of the country in case of severe events (as in 1988, 1998 and 2004). In the case of the Philippines, between 6 to 9 typhoons make landfall every year producing thousands of human losses and incalculable urban destruction; in November of 2013, typhoon Haiyan produced 6241 causalities and material damage of over 809 millions USD [see 39]. In these examples, it is crucial to be able to count with a robust system of humanitarian relief facilities that even in the worst possible scenario can provide assistance with the quickest possible response reducing the number of human loses after the occurrence of the event.

The Uncapacitated Facility Location Problem (UFL), also referred as the Simple Plant Location Problem, is one of the fundamental models in the wide spectrum of Facility Location problems [see, e.g., recent overviews presented in 23, 21]. In the classical deterministic version of the UFL one is given the set of customers, the set of locations, the facility set-up costs and the transportation costs. The goal is to define where to open facilities and how to allocate the customers to them so that the sum of set-up plus transportation costs is minimized.

In practice, it is usually the case that from the moment that the information is gathered until the moment in which the solution has to be implemented, some of the data might change with respect to the initial setting. As mentioned above, even if some (rough) idea about customers and locations is known, changes in demographic, socio-economic, or meteorological factors can lead to changes in the structure of the demand during the planning horizon, and/or the availability of a given location to host a facility (even if a facility has been already installed). This means that the solution obtained using a classical method might become infeasible and a new solution might have to be redefined from scratch. In these cases it would be better to recognize the presence of different scenarios for the data and find a 
solution comprised by first- and second-stage decisions.

Two well-known approaches to deal with uncertainty in optimization are Two-stage Stochastic Optimization (2SSO) and Robust Optimization (RO). In 2SSO [see 12] the solutions are built in two stages. In the first stage, a partial collection of decisions is defined which are later on completed (in the second stage), when the true data is revealed. Hence, the objective is to minimize the cost of the first-stage decisions plus the expected cost of the recourse (second-stage) decisions. The quality of the solutions provided by this model strongly depends on the accuracy of the random representation of the parameter values (such as probability distributions) that allow to estimate the second-stage expected cost. Nonetheless, sometimes such accuracy is not available so the use of RO models dealing with deterministic uncertainty arises as a suitable alternative [see 29, 11, 9]. On the one hand these models do not require assumptions about the distribution of the uncertain input parameters; but on the other hand, they are usually meant for calculating single-stage decisions that are immune (in a certain sense) to all possible realizations of the parameter values.

A novel alternative that combines $\mathrm{RO}$ and $2 \mathrm{SSO}$ is Two-stage Robust Optimization (2SRO); as in RO, no stochasticity of the parameters is assumed, and as in 2SSO, decisions are taken in two stages. In this case, the cost of the second-stage decision is computed by looking at the worst-case realization of the data. Therefore, the goal of 2SRO is to find a robust first-stage solution that minimizes both the first-stage cost plus the worst-case second-stage cost among all possible data outcomes. 2SRO is a rather generic classification of models; for references on different 2SRO settings we refer the reader to [8, 50].

One of the possibilities in the 2SRO framework is Recoverable Robustness [see 36]. Recalling our practical motivation, assume that the facility location and allocation policy is defined in two stages such that we are required to find a first-stage solution that should be robust against the possible realizations (scenarios) of the input data in a second stage. This means that the first-stage solution is expected to perform reasonably well, in terms of feasibility and/or optimality, for any possible realization of the uncertain parameters. An essential element of this approach is the possibility of recovering the solution built in the first stage (i.e., to modify the previously defined location-allocation policy in order to render it feasible and/or cheaper) once the uncertainty is resolved in a second stage. The recovery plan is comprised by recovery actions which are known in advance and whose costs might also depend on the possible scenario. This recovery plan is limited, in the sense that the effort needed to recover a solution may be limited algorithmically (in terms of how a solution may be modified) and economically (in terms of the total cost of recovery actions). Therefore, instead of looking for a static solution that is robust against all possible scenarios without allowing any kind of recovery [which is the case for many RO approaches, see 9], we want a solution robust enough so that it can be recovered promptly and at low cost once the uncertainty is resolved. This balance between robustness and recoverability is what defines a recoverable robust optimization problem.

With respect to the UFL, we want to find a solution whose first-stage component (opening of some facilities and allocating some customers) is implemented before the complete realization of the data. This solution can then be recovered in the second stage (to turn it into a feasible one) once the actual sets of customers and locations become available. In this case the recovery actions correspond to the opening of new facilities, the establishment of new allocations and the re-allocation of customers. 
The Recoverable Robust UFL (RRUFL) looks for a solution that minimizes the sum of the first-stage costs plus the second-stage robust recovery cost defined as the the worst case recovery cost over all possible scenarios. A formal definition of the RRUFL is given in $\$ 2.1$.

\subsection{Our Contribution and Outline of the Paper}

The contributions of this work can be summarized as follows: (i) Due to the nature of the considered uncertainty, we use a recent concept of recoverable robust optimization to formulate a Mixed Integer Programming (MIP) model that allows to derive a facility location and allocation policy composed by first- and second-stage decisions; (ii) for this novel problem we design a sophisticated exact branch-and-cut framework based on Benders decomposition which is complemented by several non-trivial enhancements; (iii) using instances from two different large classes (representing transportation and disaster management settings) we analyze in detail the characteristics of the proposed model and the obtained solutions as well as the effectiveness, behavior, and limitations of the designed approach.

In \$2 the concept of Recoverable Robustness is presented and the RRUFL is formally defined. The proposed algorithmic framework is described in \&3. The description of the benchmark instances and a detailed analysis of the computational results are presented in $\$$. Finally, conclusions and final remarks are given in $\$ 5$.

\subsection{The Uncapacitated Facility Location Problem}

It is hard to establish a single seminal work presenting the UFL, nonetheless [30] is usually regarded as the earliest work where the UFL is presented as commonly known today. We refer the reader to [18, 49] (including the references therein) for comprehensive surveys on the UFL and some of its variants.

A MIP formulation for the UFL can be given as follows. Let $R$ be the set of customers, $J$ the set of potential location of facilities, and $A$ a set of links $(i, j)$ connecting customers $i$ in $R$ with locations $j$ in $J(A \subseteq R \times J)$. The cost of opening a facility at location $j \in J$ is given by $f_{j}$, and the cost of assigning customer $i \in R$ to facility $j \in J$ using an existing link $(i, j)$ is given by $c_{i j}$. Let $\mathbf{y} \in\{0,1\}^{|J|}$ be a vector of binary variables such that $y_{j}=1$ if a facility is opened at location $j \in J$ and $y_{j}=0$ otherwise, and let $\mathbf{x} \in\{0,1\}^{|A|}$ be a vector of binary variables such that $x_{i j}=1$ if customer $i \in R$ is allocated to a facility in $j \in J$ using link $(i, j) \in A$. Using this notation, the UFL can be formulated as follows:

$$
\begin{aligned}
& O P T=\min \sum_{j \in J} f_{j} y_{j}+\sum_{(i, j) \in A} c_{i j} x_{i j} \\
& \text { s.t. } \quad \sum_{(i, j) \in A} x_{i j}=1, \quad \forall i \in R \\
& x_{i j} \leq y_{j}, \\
& \mathbf{y} \in\{0,1\}^{|J|} \text { and } \mathbf{x} \in[0,1]^{|A|} \text {. } \\
& \forall(i, j) \in A, \forall j \in J
\end{aligned}
$$

Despite its simple definition, the UFL is known to be NP-Hard [18]; however, the current advances in MIP solvers, computing machinery and the development of sophisticated preprocessing techniques allow to find optimal or nearly optimal solutions for large instances of the UFL within reasonable time. We refer to [33] for recent works on reduction procedures for the UFL. 
The incorporation of different types of uncertainty when modeling and solving the UFL is not new; in $\$ 2.2$ we will provide a brief review of Facility Location under uncertainty and compare our setting with previously proposed problems.

\section{The Recoverable Robust UFL}

In this section we present a literature review on Recoverable Robustness and formally define the RRUFL.

Recoverable Robust Optimization Recoverable Robust Optimization (RRO) was first introduced in [35, 36] as a tool for decision making under uncertainty in applications arising in the railway scheduling. In [15] and [20] one can find further applications of RRO in the context of railway scheduling.

In the last couple of years, RRO has been applied to other problems. The recoverable robust knapsack problem considering different models of uncertainty is studied in [14. Formulations and algorithms for different variants of the recoverable robust shortest path problem are given in [13]. Models, properties and exact algorithms for recoverable robust two-level network design problems are presented in [5]. A more general framework of the RRO is studied in [17] where multiple recovery stages are allowed. The authors apply this new model to timetabling and delay management applications.

Different types of uncertainty, e.g., interval, polyhedral and discrete sets, can be included in the decision process trough RRO. In this paper, we use discrete sets to model the uncertainty.

\subsection{A Formulation of the RRUFL}

As mentioned above, facility location along with the corresponding allocation decisions are typically exposed to uncertainty in different input data. As described in [43], it is possible to classify uncertainty in three categories: provider-side uncertainty, receiver-side uncertainty, and in-between uncertainty. The first corresponds to the uncertainty in facility capacity, facility reliability, facility availability, etc.; the second is related to the uncertain structure of the set of customers, customer demands, customer locations, etc.; and the third refers to the lack of complete knowledge about the transportation network topology, transportation times or costs between facilities and customers. The proposed recoverable robust UFL model is a general approach and it can address situations in which uncertainty may be present in any of these three categories.

Suppose we are given an instance of the UFL in which uncertainty is present in the set of customers $R$, the set of locations $J$, the set of allocation links $A$ and the corresponding setup and allocation costs. Such application might arise, for instance, in the event of natural disasters. In these cases it can be very hard to estimate in advance (i) which areas will require humanitarian relief, (ii) where the emergency facilities (e.g., Red Cross facilities) can be located and (iii) how the damaged areas can be reached by the emergency brigades coming from the installed facilities. Therefore, instead of dealing with deterministic sets $R$, $J$ and $A$ we are given a finite set $K$ of discrete scenarios such that each scenario $k \in K$

is characterized by its own sets $R^{k}, J^{k}$ and $A^{k}$ and also by the corresponding set-up and allocation costs. 
Formally, let $K$ be a set of scenarios representing possible realizations of the problem data, more precisely, for a given $k \in K$ : let $R^{k}$ be the set of customers that require the service if scenario $k$ is realized; let $J^{k}$ be the set of locations where facilities can be opened if scenario $k$ is realized; and let $A^{k}$ be the set of links that can be used if scenario $k$ is realized. We define $R^{0}=\bigcup_{k \in K} R^{k}$ as the set of potential customers, $J^{0}=\bigcup_{k \in K} J^{k}$ as the set of potential locations and $A^{0}=\bigcup_{k \in K} A^{k}$ as the set of potential connections. We assume that the classical UFL has at least one feasible solution for $R^{0}, J^{0}$ and $A^{0}$, and that each customer $i \in R^{k}$ can be reached by some link from $A^{k}$.

The decision maker faces a two-stage decision: she/he needs to define a first-stage plan (to open some facilities and to allocate some customers to these open facilities) without knowing in advance the actual data that will be revealed. Once the actual information is available in a second stage (i.e., a single $k \in K$ and its corresponding $R^{k}, J^{k}$ and $A^{k}$ ) additional decisions can be taken in order to recover the first-stage plan and turn it into a feasible solution for the revealed data. A second-stage decision is said to be feasible if for all $k \in K$ each customer $i \in R^{k}$ is allocated to one installed facility in $j \in J^{k}$ and the allocation link is operational, i.e., $(i, j) \in A^{k}$. These second-stage decisions consist of (i) the opening of new facilities, (ii) the allocation of customers to facilities that are either opened in the second-stage or were opened in the first-stage, and (iii) the re-allocation of customers that were allocated in the first-stage to facilities that are actually not available in the realized scenario.

In Figure 1(a) an instance of the RRUFL with set of facilities $J^{0}=\{A, B, C\}$, set of customers $R^{0}=\{1,2,3,4\}$ and with two scenarios is shown. Scenario $k=1$ is given by $R^{1}=\{1,3,4\}, J^{1}=\{A, B\}, A^{1}=\{(1, A),(1, b),(3, A),(4, B)\}$, and scenario $k=2$ is given by $R^{2}=\{2,3,4\}, J^{2}=\{B, C\}, A^{2}=\{(2, B),(4, B),(3, C)\}$. In the first stage, allocation and facility set-up costs are 1 and 2 , respectively. In the second stage, allocation and set-up costs are 1.5 and 3, respectively, the cost of re-allocating a customer is 2 and the penalty for a facility opened at a non-available site is 3.5. A first-stage solution is shown in Figure 1(b); a facility at site $A$ is opened, customers 1 and 3 are allocated to it and the total cost is: 2 (one opening) $+1+1$ (two allocations) $=4$. For this given first-stage decision, we present in Figure 1(c) the optimal second-stage solution in case scenario $k=1$ is realized: a facility at site $B$ has to be installed while the facility at $A$ remains open, customers 1 and 3 keep their allocations while customer 4 is allocated to the facility in $B$; so the second-stage cost is: 3 (one opening) +1.5 (one allocation) $=4.5$. The optimal second-stage solution in case scenario $k=2$ is realized is shown in Figure $1(\mathrm{~d})$; facilities at $B$ and $C$ have to be installed while the facility at $A$ becomes unavailable, customers 2 and 4 are allocated to the facility at $B$, while customer 3 has to be re-allocated to the facility in $C$; the cost is: $3+3$ (two opening $)+1.5+1.5$ (two allocations $)+2$ (one re-allocation $)+3.5$ (one penalty $)=$ 14.5. Therefore, in the worst case, the overall cost of establishing this first-stage solution and recover it in the second stage is given as $\max \{4+4.5,4+14.5\}=18.5$. Our goal will be to find the optimal first-stage decision, so that in the worst-case total cost of the firstand second-stage is minimized. For this example, the optimal first-stage solution is defined by the installation of a facility in $B$ and the allocation of 4 to it; this solutions induces a first-stage cost of 3 and worst case second stage cost of 6 , yielding a total cost of 9 .

MIP Formulation In the first stage, decisions are modeled as follows: let $\mathbf{y}^{0} \in\{0,1\}^{\left|J^{0}\right|}$ 


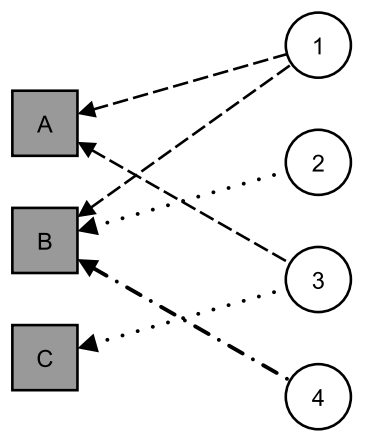

(a) Instance of RRUFL

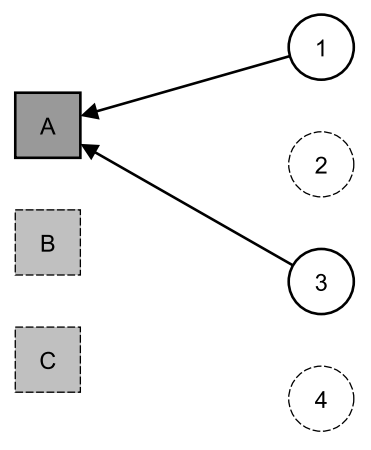

(b) A 1st-stage solution

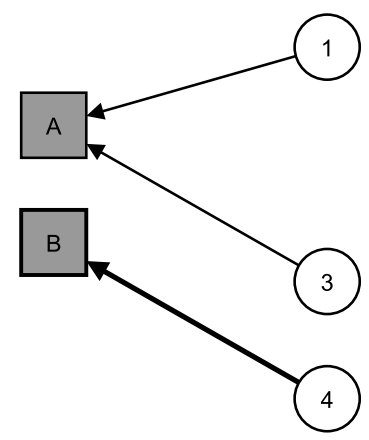

(c) Solution for $k=1$

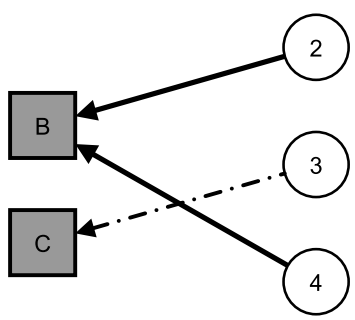

(d) Solution for $k=2$

Figure 1: Example of an instance and first- and second-stage solutions for the RRUFL.

be a vector of binary variables such that $y_{j}^{0}=1$ if a facility is opened at location $j \in J^{0}$ in the first stage (at cost $f_{j}^{0}$ ) and $y_{j}^{0}=0$ otherwise; let $\mathbf{x}^{0} \in\{0,1\}^{\left|A^{0}\right|}$ be a vector of binary variables such that $x_{i j}^{0}=1$ if the $\operatorname{link}(i, j) \in A^{0}$ is used to allocate customer $i \in R^{0}$ to the facility at $j \in J^{0}$ (at $\operatorname{cost} c_{i j}^{0}$ ) and $x_{i j}^{0}=0$ otherwise. For a given scenario $k \in K$, secondstage decisions are defined as follows: let $\mathbf{y}^{k} \in\{0,1\}^{\left|J^{k}\right|}$ be a vector of binary variables such that $y_{j}^{k}=1$ if a facility is opened at location $j \in J^{k}$ in the second stage (at cost $f_{j}^{k}$ ) and $y_{j}^{k}=0$ otherwise; let $\mathbf{x}^{k} \in\{0,1\}^{\left|A^{k}\right|}$ be a vector of binary variables such that $x_{i j}^{k}=1$ if the link $(i, j) \in A^{k}$ is used to allocate customer $i \in R^{k}$ to the facility at $j \in J^{k}$ (at cost $c_{i j}^{k}$ ) and $x_{i j}^{k}=0$ otherwise; and let $\mathbf{z}^{k} \in\{0,1\}^{\left|A^{k}\right|}$ be a vector of binary variables such that $z_{i l}^{k}=1$ if the link $(i, l) \in A^{k}$ is used to re-allocate customer $i \in R^{k}$ to the facility at $l \in J^{k}$ (at cost $\left.r_{i l}^{k}\right)$ and $z_{i l}^{k}=0$ otherwise. If a facility is installed in the first stage at a given location $j \in J^{0}$ $\left(y_{j}^{0}=1\right)$ and this location is available if scenario $k$ is realized in a second stage $\left(j \in J^{k}\right)$, then this facility remains open and no extra cost is incurred; if the location is not available in the second stage $\left(j \in J^{0} \backslash J^{k}\right)$, then a penalty $p_{j}^{k}$ must be paid.

With this definition of variables, a first-stage solution is a pair $\left.\left(\mathbf{x}^{0}, \mathbf{y}^{0}\right) \in\{0,1\}\right|^{\left|A^{0}\right|+\left|J^{0}\right|}$ satisfying

$$
\begin{aligned}
& x_{i j}^{0} \leq y_{j}^{0}, \quad \forall(i, j) \in A^{0} \\
& \sum_{(i, j) \in A^{0}} x_{i j}^{0} \leq 1, \quad \forall i \in R^{0} \text {. }
\end{aligned}
$$

Given a first-stage solution $\left(\mathbf{x}^{0}, \mathbf{y}^{0}\right)$ and a scenario $k \in K$, the recovery cost is the minimum total cost $\rho\left(\mathbf{x}^{0}, \mathbf{y}^{0}, k\right)$ of the second-stage recovery actions $\left(\mathbf{x}^{k}, \mathbf{y}^{k}, \mathbf{z}^{k}\right)$ needed to render the solution feasible. Hence, $\rho\left(\mathbf{x}^{0}, \mathbf{y}^{0}, k\right)$ is found by solving the following recovery problem: 


$$
\begin{aligned}
& \rho\left(\mathbf{y}^{\mathbf{0}}, \mathbf{x}^{\mathbf{0}}, k\right)=\min \sum_{j \in J^{k}} f_{j}^{k}\left(y_{j}^{k}-y_{j}^{0}\right)+\sum_{(i, j) \in A^{k}} c_{i j}^{k} x_{i j}^{k}+\sum_{(i, l) \in A^{k}} r_{i l}^{k} z_{i l}^{k}+\sum_{j \in J^{0} \backslash J^{k}} p_{j}^{k} y_{j}^{0} \\
& \text { s.t. } \quad \sum_{(i, j) \in A^{0}} x_{i j}^{0}+\sum_{(i, j) \in A^{k}} x_{i j}^{k}=1, \quad \forall i \in R^{k} \\
& \sum_{(i, j) \in A^{0} \backslash A^{k}} x_{i j}^{0} \leq \sum_{(i, l) \in A^{k}} z_{i l}^{k}, \quad \forall i \in R^{k} \\
& x_{i j}^{k}+z_{i j}^{k} \leq y_{j}^{k}, \quad \forall(i, j) \in A^{k}, \forall i \in R^{k} \\
& y_{j}^{0} \leq y_{j}^{k}, \quad \forall j \in J^{k} \\
& \mathbf{y}^{\mathbf{k}} \in\{0,1\}^{\left|J^{k}\right|}, \mathbf{x}^{\mathbf{k}} \in\{0,1\}^{\left|A^{k}\right|},\left.\mathbf{z}^{\mathbf{k}} \in\{0,1\}\right|^{\left|A^{k}\right|} .
\end{aligned}
$$

Objective function ( $\mathrm{R.1}$ ) is comprised by the set-up cost of facilities in the second-stage $\left(\sum_{j \in J^{k}} f_{j}^{k}\left(y_{j}^{k}-y_{j}^{0}\right)\right)$, the allocation cost in the second-stage $\left(\sum_{(i, j) \in A^{k}} c_{i j}^{k} x_{i j}^{k}\right)$, the cost of re-allocating customers $\left(\sum_{(i, l) \in A^{k}} r_{i l}^{k} z_{i l}^{k}\right)$, and the total penalty paid by those facilities opened in the first stage that can not operate if scenario $k \in K$ is realized $\left(\sum_{j \in J^{0} \backslash J^{k}} p_{j}^{k} y_{j}^{0}\right)$. Constraints $(\mathrm{R} .2)$ state that a customer is either allocated in the first stage $\left(\sum_{(i, j) \in A^{0}} x_{i j}^{0}\right)$ or in the second-stage $\left(\sum_{(i, j) \in A^{k}} x_{i j}^{k}\right)$. Constraints (R.3) model the fact that if a customer $i \in R^{k}$ has been allocated in the first-stage to a facility $j \in J^{0}$ by means of a link $(i, j) \in A^{0} \backslash A^{k}$ then it has to be re-allocated to another facility $l \in J^{k}$ through a link $(i, l)$ available in the second-stage $\left(\sum_{(i, l) \in A^{k}} z_{i l}^{k}\right)$. Constraints (R.4) impose that if a customer is allocated or re-allocated to a facility $j \in J^{k}$, then that facility has to be available and reachable in the second-stage. The fact that a facility that has been opened in the first stage should remain opened in the second stage is modeled by (R.5). The nature of the variables is imposed in (R.6) (note that one can also relax the integrality constraints for $\mathbf{x}^{k}$ and $\mathbf{z}^{k}, \forall k \in K$ ).

For a given first-stage solution $\left(\mathbf{x}^{0}, \mathbf{y}^{0}\right)$ the robust recovery cost $R\left(\mathbf{x}^{0}, \mathbf{y}^{0}\right)$ corresponds to the maximum recovery cost among all $k \in K$, i.e.,

$$
R\left(\mathbf{x}^{0}, \mathbf{y}^{0}\right)=\max _{k \in K} \rho\left(\mathbf{x}^{\mathbf{0}}, \mathbf{y}^{\mathbf{0}}, k\right) .
$$

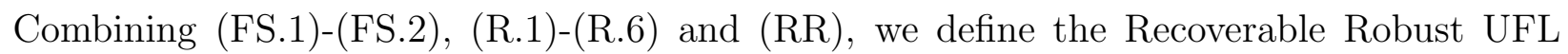
problem (RRUFL) as

$$
\begin{aligned}
& O P T_{\mathcal{R} R}=\min \sum_{j \in J^{0}} f_{j}^{0} y_{j}^{0}+\sum_{(i, j) \in A^{0}} c_{i j}^{0} x_{i j}^{0}+R\left(\mathbf{x}^{0}, \mathbf{y}^{0}\right) \\
& \text { s.t. }\left(\text { FS.1)- } \text { FS.2 }, \text { R.2 } 2 \text { R. } 6 \text { and }\left(\mathbf{x}^{0}, \mathbf{y}^{0}\right) \in\{0,1\}\left|A^{0}\right|+\left|J^{0}\right| .\right.
\end{aligned}
$$

In the proposed formulation of the RRUFL we impose that each customer $i \in R^{k}$ has to be assigned (or re-assigned) to exactly one available facility $j \in J^{k}$ for any given $k \in K$. It is possible to relax this and, instead, impose a penalty, say $t_{i}^{k}$, if customer $i \in R^{k}$ is not served by any facility if scenario $k$ is realized. This can be done by introducing a dummy facility $\pi^{k}$ with a set-up cost equal to 0 and connecting it to every customer $i \in R^{k}$ with an allocation (and re-allocation) $\operatorname{cost} c_{i \pi}^{k}=r_{i \pi}^{k}=t_{i}^{k}$. 
In many applications it is natural to think that whichever decision we take in the future it will be more expensive than if it would have been taken at present. For instance, opening a facility at a given location is likely to be more expensive later on in the planning horizon than now $\left(f_{j}^{k} \geq f_{j}^{0}\right)$. Likewise, an agreement between a depot (facility) and a customer is expected to have better conditions (for one of the two parties at least) if it is established earlier than if it is defined when the market conditions have evolved $\left(c_{i j}^{k} \geq c_{i j}^{0}\right)$. Furthermore, it is also natural to think that if an already agreed pact between a depot and a customer is forced to be changed (e.g., because no allocation link is available between them), this will entail an additional re-allocation cost possibly higher than the original one $\left(r_{i l}^{k} \geq c_{i j}^{0}\right.$, for all $\left.l \in J^{k}\right)$.

An optimal first-stage solution $\left(\mathbf{x}^{0}, \mathbf{y}^{0}\right)$ is robust because, regardless which scenario occurs, it guarantees that the second-stage actions will be efficient (due to the minimization of the worst case) and easy to implement (because only a simple UFL has to be solved). Hence, the more scenarios we take into consideration to find $\left(\mathbf{x}^{0}, \mathbf{y}^{0}\right)$, the more robust the solution is; because we are foreseeing more possible states of the future uncertainty. Unlike common approaches of RO that protect solutions against perturbations in parameters as costs or demands, our approach also hedges against uncertainty in the very topology of the network. Likewise, a first-stage solution is recoverable, or possesses recoverability, because it can become feasible in a second stage by means of second-stage actions.

The Robust UFL without Recovery To assess the effectiveness and benefits of the RRULF, we also introduce another natural, but more conservative, model. Assume a decision-making context equivalent to the one taken into account before. Consider a model in which first-stage decisions are comprised only by $\mathbf{y}^{0}$ and second-stage decisions only by $\mathbf{x}^{k}, \forall k \in K$. This is, an 2SRO model in which facilities can be opened only in the first stage and allocations can be decided only in the second stage. We will refer to this new problem simply as Robust Uncapacitated Facility Location without Recovery (RUFL). This alternative model lacks the concept of recoverability since the solution cannot be intrinsically changed: no new facility can be opened and there is no need to re-allocate any customer in the second stage. Therefore, the solutions of such model although possibly more robust (since they are more conservative) are expected to be more expensive, either because unnecessarily many facilities have to be opened in the first stage or because the second-stage allocation costs are considerably higher than those of the first stage. If we consider again the instance in Figure 1(a), one can easily see that for this new model the optimal (and only feasible) first-stage solution would be given by the installation of facilities in $A, B$ and $C$ (with a cost of 6 ). In both $k=1$ and $k=2$ the optimal second-stage cost would be 8 . This leads to a total cost equal to $6+\max \{8,8\}=14$, which is more than the cost of the optimal solution of the RRUFL which is 9 .

\subsection{The RRUFL and Previously Proposed Problems}

Already in the 70's efforts were devoted to provide both theoretical and algorithmic contributions on Stochastic UFL. In [44] one can find an excellent review on Facility Location under uncertainty, describing contributions not only from the stochastic but also from the RO perspective. More recent references to Facility Location under uncertainty include [45, 7, 46, 19, 43, 2, 1, 25, 4, 3, 26] and [34]. 
Our definition of the RRUFL, as well as the algorithmic framework described later, spans different possible cases of uncertainty in Facility Location. Some of them have been already addressed in the literature by the use of stochastic and robust two-stage models.

For instance if $J^{k}=J^{0}$ and $A^{k}=A^{0}, \forall k \in K$, then we are only addressing uncertainty in the set of customers and, eventually, in the second-stage costs. A 2SSO approach for this problem has been considered in [41, where approximation algorithms have been proposed. In [45, 19, 43] and [34, uncertainty has been addressed only in the set of locations $\left(R^{k}=R^{0}\right.$ and $\left.A^{k}=A^{0}, \forall k \in K\right)$. As stressed by the authors, this model is suitable for applications where facilities might become unavailable in a second stage due to disruptions caused by natural disasters, terrorists attacks or labor strikes [see 19]. These papers share two important features. First, uncertainty is tackled by means of 2SSO since probabilities of facility failure are known in advance for each scenario. Second, a user is assigned to a so-called primary facility that will serve it under normal circumstances, as well as to a set of ordered backup facilities such that the first of them that is available will serve the customer when the primary is not available [see 45]. This second feature cannot be included in our framework without introducing additional binary variables; nonetheless decision-maker preferences about the re-allocation of a customer in case the originally assigned facility fails can be incorporated by a proper definition of the re-allocation second-stage costs.

A third case is the one where only connections are subject to uncertainty $\left(R^{k}=R^{0}\right.$ and $\left.J^{k}=J^{0}, \forall k \in K\right)$. A 2SRO model of this case is studied in [28] where the relevance of such a model of uncertainty is emphasized in the context of response planning after disasters.

\section{Algorithmic Framework}

Note that formulation (1)-(2) has a polynomial number of variables and constraints with respect to $\left|R^{0}\right|,\left|A^{0}\right|$ and $|K|$. Therefore it can be solved directly (as a compact model) through any state-of-the-art MIP solver (e.g., CPLEX). However, as we will show later, when large realistic instances have to be solved, the direct use of solvers turns out to be impractical.

Model (1)-(2) is a natural candidate to be solved by means of a Benders-like decomposition approach: the first-stage variables $\left(\mathbf{x}^{0}, \mathbf{y}^{0}\right)$ are incorporated in the master problem (MP) and the second-stage variables $\left(\mathbf{x}^{k}, \mathbf{y}^{k}, \mathbf{z}^{k}\right)$ are projected out and replaced by a single variable $\omega$ representing the robust recovery cost, for a given $\left(\tilde{\mathbf{x}}^{0}, \tilde{\mathbf{y}}^{0}\right)$, that is computed by solving $|K|$ slave problems (SPs). Thus, the objective function (1) becomes $O P T_{\mathcal{R} R}=\min \sum_{j \in J^{0}} f_{j}^{0} y_{j}^{0}+\sum_{(i, j) \in A^{0}} c_{i j}^{0} x_{i j}^{0}+\omega$, where $\omega \geq \rho\left(\mathbf{x}^{0}, \mathbf{y}^{0}, k\right), \forall k \in K$. Hence, for each given value of $\left(\mathbf{x}^{0}, \mathbf{y}^{0}, k\right), \omega$ can be computed by independently solving $|K|$ problems R.1 - R.6.

In our framework we refrain from the traditional implementation of Benders decomposition, given the drawback that several MIP problems (MP and SPs) need to be solved at each iteration in order to obtain a single Benders-cut. Nowadays most of MIP optimization suites provide branch-and-cut frameworks supported by the use of callbacks. These callbacks allow for the Benders decomposition to be transformed into a pure branch-and-cut approach. The implementation works as follows: Benders cuts are added to the model as valid lower-bounds on $\omega$ each time a potential (fractional) solution of the MP is found by means of solving a Linear Programming (LP) problem in a given node of the enumeration 
tree. This technique exploits the benefits of the decomposition allowing to implement additional methods for heuristically finding more cuts and/or for strengthening the obtained ones. That way, both, the speed and the convergence of the algorithm can be improved [see, e.g., recent implementations of [37, 40].

Basic Separation of $\boldsymbol{L}$-shaped and Integer $\boldsymbol{L}$-shaped Cuts In our approach, a valid lower bound on $\omega$ is iteratively imposed by means of $L$-shaped and integer $L$-shaped cuts [see 48, 31]. For a given first-stage solution, the second-stage problem can be decomposed into $|K|$ independent problems: dual variables of the LP-relaxations of these SPs yield $L$-shaped cuts that are added to the MP while integer solutions of the SPs yield integer $L$-shaped cuts.

At a given node of the enumeration tree, let $\left(\tilde{\mathbf{x}}^{0}, \tilde{\mathbf{y}}^{0}\right)$ be a first-stage solution satisfying $($ FS.1 $)-($ FS.2 $)$ and let $\tilde{\omega}$ be the current value of variable $\omega$. For a given $k \in K$, the dual of (R.1)-(R.6) after removing the integrality constrains can be formulated as

$$
\begin{aligned}
& \max \sum_{i \in R^{k}}\left[\alpha_{i}\left(1-\sum_{(i, j) \in A^{0}} \tilde{x}_{i j}^{0}\right)+\gamma_{i}\left(\sum_{(i, j) \in A^{0} \backslash A^{k}} \tilde{x}_{i j}^{0}\right)\right]+\sum_{j \in J^{k}}\left(\epsilon_{j}-f_{j}^{k}\right) \tilde{y}_{j}^{0}+\sum_{j \in J^{0} \backslash J^{k}} p_{j}^{k} \tilde{y}_{j}^{0} \\
& \text { s.t. } \alpha_{i}-\delta_{i j} \leq c_{i j}^{k}, \quad \forall(i, j) \in A^{k}, \forall i \in R^{k} \\
& \quad \gamma_{i}-\delta_{i l} \leq r_{i l}^{k}, \quad \forall(i, l) \in A^{k}, \forall i \in R^{k} \\
& \quad \epsilon_{j}+\sum_{(i, j) \in A^{k}} \delta_{i j} \leq f_{j}^{k}, \quad \forall j \in J^{k} \\
& \quad(\boldsymbol{\alpha}, \boldsymbol{\gamma}, \boldsymbol{\delta}, \boldsymbol{\epsilon}) \geq \mathbf{0}
\end{aligned}
$$

where $\boldsymbol{\alpha}, \boldsymbol{\gamma}, \boldsymbol{\delta}$ and $\boldsymbol{\epsilon}$ correspond to the dual variables of constraints (R.2), (R.3), (R.4) and (R.5), respectively. Let $(\tilde{\boldsymbol{\alpha}}, \tilde{\boldsymbol{\gamma}}, \tilde{\boldsymbol{\delta}}, \tilde{\boldsymbol{\epsilon}})$ be an optimal solution to (D.1)-(D.5) with optimal value $\tilde{\rho}^{k}$. Following the LP-duality theory, an L-shaped (optimality) cut is given by

$$
\omega \geq \sum_{i \in R^{k}}\left[\tilde{\alpha}_{i}\left(1-\sum_{(i, j) \in A^{0}} x_{i j}^{0}\right)+\tilde{\gamma}_{i}\left(\sum_{(i, j) \in A^{0} \backslash A^{k}} x_{i j}^{0}\right)\right]+\sum_{j \in J^{k}}\left(\tilde{\epsilon}_{j}-f_{j}^{k}\right) y_{j}^{0}+\sum_{j \in J^{0} \backslash J^{k}} p_{j}^{k} y_{j}^{0},
$$

which is added to the model if $\tilde{\omega}<\tilde{\rho}^{k}$. Note that an $L$-shaped cut (LS) can be found regardless of $\left(\tilde{\mathbf{x}}^{0}, \tilde{\mathbf{y}}^{0}\right)$ being integer.

Now suppose that $\left(\tilde{\mathbf{x}}^{0}, \tilde{\mathbf{y}}^{0}\right)$ is integer. If there is no $k \in K$ with $\tilde{\omega}<\tilde{\rho}^{k}$, then one can attempt to find integer $L$-shaped cuts [see 31]. For a given $k \in K$, let $\check{\rho}^{k}$ be the optimal value of (R.1)-(R.6) (preserving the integrality constraints), if $\tilde{\omega}<\check{\rho}^{k}$, then the following valid inequality can be added to the MP,

$$
\omega \geq \check{\rho}^{k}\left(\sum_{(i, j) \in \mathcal{A}^{k}}\left(x_{i j}^{0}-1\right)-\sum_{(i, j) \in A^{k} \backslash \mathcal{A}^{k}} x_{i j}^{0}+\sum_{j \in \mathcal{J}^{k}}\left(y_{j}^{0}-1\right)-\sum_{j \in J^{k} \backslash \mathcal{J}^{k}} y_{j}^{0}+1\right),
$$

where $\mathcal{A}^{k}=\left\{(i, j) \in A^{k} \mid \tilde{x}_{i j}^{0}=1\right\}$ and $\mathcal{J}^{k}=\left\{j \in J^{k} \mid \tilde{y}_{j}^{0}=1\right\}$ are the index sets of the links $(i, j) \in A^{k}$ and locations $j \in J^{k}$ chosen in the first stage, respectively. 


\subsection{Strengthening and Calculating Additional L-shaped Cuts}

In the following we will describe different enhancements that we have incorporated into our algorithmic framework.

Scenario Sorting Formally speaking, when separating (LS) cuts we only need to add the cut associated with the worst-case scenario $k^{*}=\arg \max _{k \in K}\left(\tilde{\rho}^{k}\right)$ for a given $\left(\tilde{\mathbf{x}}^{0}, \tilde{\mathbf{y}}^{0}\right)$. However this entails an important disadvantage: exactly $|K|$ LPs and/or ILPs have to be solved to optimality, and only a single cut is generated out of this eventually large computational effort.

In order to overcome the above described drawback we have designed a strategy that first dynamically sorts scenarios according to the information available from the previous iterations and then attempts to add not a single but many potentially good cuts. We first note that as long as $\tilde{\omega}<\tilde{\rho}^{k}$, one can add an (LS) cut. Secondly, it is intuitive to think that for a given instance there is a subset of scenarios that systematically induce violated cuts, while another subset of scenarios rarely do so. Therefore, on the basis of the cut violation values, defined as $\tilde{\rho}^{k}-\tilde{\omega}$, one can dynamically update an ordered list of scenarios

$\bar{K}=\left[\bar{k}_{1}, \bar{k}_{2}, \ldots, \bar{k}_{K}\right]$, placing in the first positions those scenarios that consistently induce large cut violation and at the end those that rarely satisfy $\tilde{\omega}<\tilde{\rho}^{k}$.

In our strategy we apply learning mechanisms to identify $\bar{K}$ and prioritize the search of violated $L$-shaped cuts using the first elements of the list until a pre-fixed number $M A X_{\text {cut }} \leq$ $|K|$ of violated cuts has been found or a pre-fixed number $M A X_{\text {fail }} \leq|K|$ of failed attempts has been reached.

In Algorithm 1 we present the general scheme of the separation of $L$-shaped cuts using the scenario sorting strategy. For each scenario $k \in K$, the value $f r e q[k]$ accumulates the number of separation calls in which we have solved the corresponding SP. Likewise, the value viol $[k]$ is a cumulative cut violation value of scenario $k$, over all previous separation calls. In Step 1 the list $\bar{K}$ is created and its elements are sorted in decreasing order with respect to viol $[k] /$ freq $[k]$, which represents the average violation that each scenario has induced in the previous iterations. In loop 3,12 the $L$-shaped cuts are added: in line 4 the first scenario in the list $\bar{K}$ is taken and removed; the $k$-th SP is solved in line 5 ; both vectors needed to sort scenarios are updated in line 6, if the solution of the SP induces a violated cut (line 7) then the corresponding inequality is added in line 8 and the counter of added cuts is increased (line 9); if no violated cut is generated, the corresponding counter is increased in line 11.

In our default implementation (and after parameter tuning), we have set $M A X_{\text {cut }}=$ $0.25 \times|K|$ and $M A X_{\text {fail }}=0.25 \times|K|$.

Dual Lifting Clearly, the strength of the generated $L$-shaped cuts will strongly influence the performance of the algorithm; the stronger they are, the less MP iterations (hence, the less explored nodes in the enumeration tree) are needed. In this paper we use a recently proposed technique to strengthen $L$-shaped cuts [see 37]. In contrast to other approaches for generating stronger cuts [see, e.g., 38], this heuristic method does not require to solve any additional LP problem and the strengthening process can be performed in linear time (with respect to the number of variables).

Let $\left(\tilde{\mathbf{x}}^{0}, \tilde{\mathbf{y}}^{0}\right)$ be a pair satisfying $(\overline{F S .1})-(\overline{F S .2}), \tilde{\omega}$ the current value of variable $\omega$, and $(\tilde{\boldsymbol{\alpha}}, \tilde{\boldsymbol{\gamma}}, \tilde{\boldsymbol{\delta}}, \tilde{\boldsymbol{\epsilon}})$ an optimal solution to (D.1)-(D.5) that satisfies $\tilde{\omega}<\tilde{\rho}^{k}$. The scheme to strengthen the corresponding $L$-shaped cut is the following: (i) If for customer $i \in R^{k}$ we have $\sum_{(i, j) \in A^{0}} \tilde{x}_{i j}^{0}=$ 


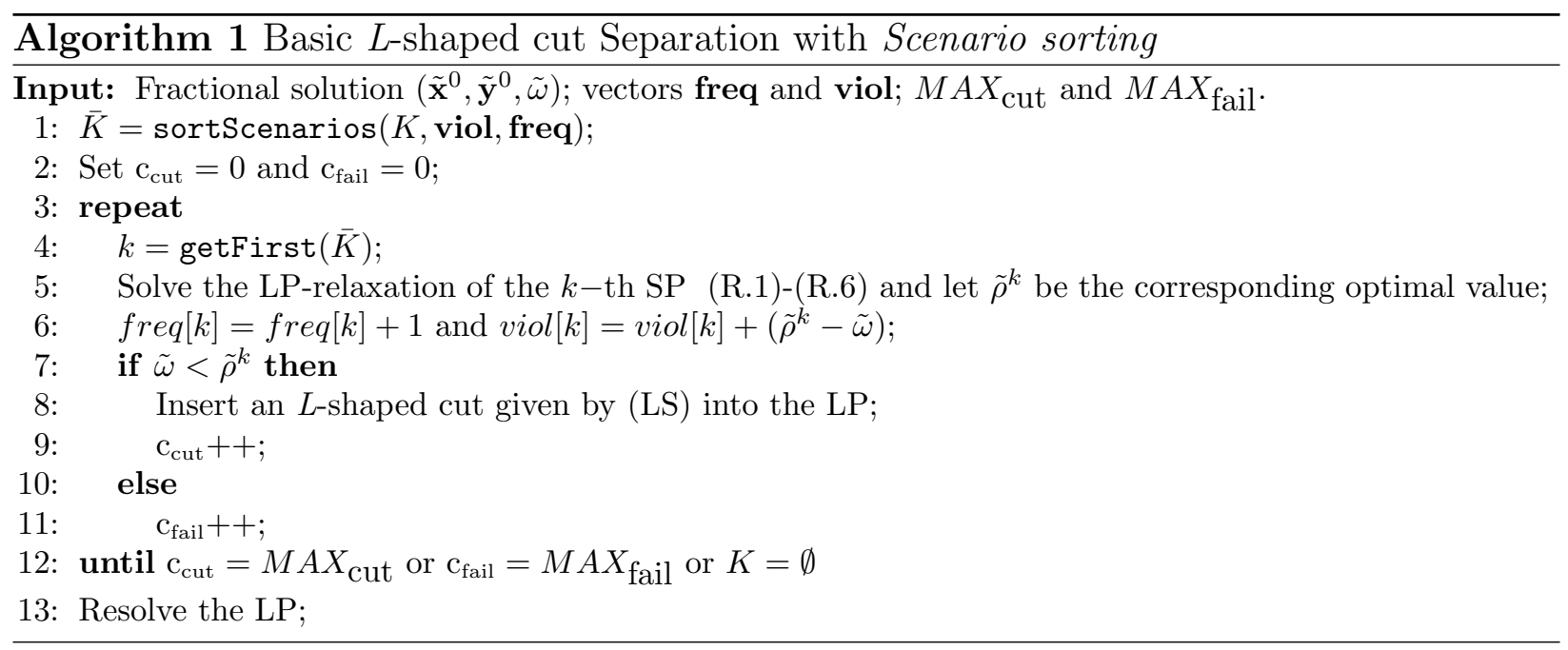

1, then the corresponding dual variable $\alpha_{i}$ does not appear in (D.1). (ii) If for customer $i \in R^{k}$ we have $\sum_{(i, j) \in A^{0} \backslash A^{k}} \tilde{x}_{i j}^{0}=0$, then the corresponding dual variable $\gamma_{i}$ does not appear in D.1. (iii) If for a facility $j \in J^{k}$ we have $\tilde{y}_{j}^{0}=0$, then the corresponding dual variable $\epsilon_{j}$ does not appear in (D.1). (iv) Moreover, variables $\boldsymbol{\delta}$ do not appear in the objective (D.1) neither. On the basis of (i)-(iv) we observe that we deal with a highly degenerate LP and one can expect that the optimal solutions to (D.2)-(D.4) usually produce positive slacks (typically, an LP solver will fix the associated dual variables to zero). The idea is now to produce another LP optimal solution of the dual SP such that these slacks are reduced to zero. Therefore, the values of the dual coefficients in (LS) will be lifted as follows:

$$
\begin{aligned}
& \check{\alpha}_{i}= \begin{cases}\tilde{\alpha}_{i} & \text { if } \sum_{(i, j) \in A^{0}} \tilde{x}_{i j}^{0}<1 \\
\min _{(i, j) \in A^{k}}\left\{c_{i j}^{k}+\tilde{\delta}_{i j}\right\} & \text { otherwise }\end{cases} \\
& \check{\gamma}_{j}= \begin{cases}\tilde{\gamma}_{j} & \text { if } \sum_{(i, j) \in A^{0} \backslash A^{k}} \tilde{x}_{i j}^{0}>0 \\
\min _{(i, j) \in A^{k}}\left\{r_{i j}^{k}+\tilde{\delta}_{i j}\right\} & \text { otherwise }\end{cases} \\
& \check{\epsilon}_{j}= \begin{cases}\tilde{\epsilon}_{j} & \text { if } \tilde{y}_{j}^{0}>0 \\
f_{j}^{k}-\sum_{(i, j) \in A^{k}} \tilde{\delta}_{i j} & \text { otherwise }\end{cases}
\end{aligned}
$$

This is why we refer to this procedure as dual lifting. If $\check{\alpha}_{i}>\tilde{\alpha}_{i}, \check{\gamma}_{j}>\tilde{\gamma}_{j}$ or $\check{\epsilon}_{j}>\tilde{\epsilon}_{j}$ for at least one $i \in R^{k}$ or $j \in J^{k}$, respectively, then the lifted $L$-shaped cut is given by

$$
\omega \geq \sum_{i \in R^{k}}\left[\check{\alpha}_{i}\left(1-\sum_{(i, j) \in A^{0}} x_{i j}^{0}\right)+\check{\gamma}_{i}\left(\sum_{(i, j) \in A^{0} \backslash A^{k}} x_{i j}^{0}\right)\right]+\sum_{j \in J^{k}}\left(\check{\epsilon}_{j}-f_{j}^{k}\right) y_{j}^{0}+\sum_{j \in J^{0} \backslash J^{k}} p_{j}^{k} y_{j}^{0} .
$$

Lemma 1 ([37]). The lifted L-shaped cuts (l-LS) are valid and strictly stronger than the standard L-shaped cuts (LS). 
From the algorithmic point of view, to apply this approach one simply has to insert a cut of type $(l-\mathrm{LS})$ instead of one of type $(\mathrm{LS})$ in line 8 of Algorithm 1 .

We finally point out that similar procedures are used for stabilizing column generation approaches [32, see, e.g.,].

Zero-half- $\boldsymbol{L}$-shaped Cuts Zero-half cuts are a subclass of rank-1 Chvátal-Gomory cuts with multipliers restricted to $\left\{0, \frac{1}{2}\right\}$ [16]. They play an important role in polyhedral theory, and nowadays they are also incorporated in major MIP solvers. Instead of using a generic zero-half cut generation [see, e.g., 6], we impose zero-half cuts in combination with the learning mechanisms introduced in the previous section. To this end, for a given $k \in K$, observe that by reordering terms, an arbitrary $(\overline{\mathrm{LS}})$ or $(l$-LS can be written as

$$
\omega \geq \Lambda\left(\overline{\boldsymbol{\xi}}^{k}\right)+\sum_{(i, j) \in A^{0}} \bar{\xi}_{i j}^{k} x_{i j}^{0}+\sum_{j \in J^{0}} \bar{\epsilon}_{j}^{k} y_{j}^{0},
$$

where $\Lambda\left(\overline{\boldsymbol{\xi}}^{k}\right)$ is a constant value and $\overline{\boldsymbol{\xi}}^{k}$ and $\overline{\boldsymbol{\epsilon}}^{k}$ are the corresponding condensed dual multipliers. Now, let us consider two scenarios $k_{1}$ and $k_{2}$ inducing cuts $(l$-LS $)$ in a given node of the search tree and such that all coefficients of (3) are integer for $k_{1}$ and $k_{2}$ (with at least one odd value). By first multiplying each coefficient of the two induced cuts by $1 / 2$ and then summing the two resulting inequalities, we get:

$$
\omega \geq \frac{1}{2}\left(\Lambda\left(\overline{\boldsymbol{\xi}}^{k_{1}}\right)+\Lambda\left(\overline{\boldsymbol{\xi}}^{k_{2}}\right)\right)+\sum_{(i, j) \in A^{0}} \frac{1}{2}\left(\bar{\xi}_{i j}^{k_{1}}+\bar{\xi}_{i j}^{k_{2}}\right) x_{i j}^{0}+\sum_{j \in J^{0}} \frac{1}{2}\left(\bar{\epsilon}_{j}^{k_{1}}+\bar{\epsilon}_{j}^{k_{2}}\right) y_{j}^{0} .
$$

By rounding up the constant term and each of the coefficients of the above inequality, we get the following zero-half cut:

$$
\omega \geq\left\lceil\frac{1}{2}\left(\Lambda\left(\overline{\boldsymbol{\xi}}^{k_{1}}\right)+\Lambda\left(\overline{\boldsymbol{\xi}}^{k_{2}}\right)\right)\right\rceil+\sum_{(i, j) \in A^{0}}\left\lceil\frac{1}{2}\left(\bar{\xi}_{i j}^{k_{1}}+\bar{\xi}_{i j}^{k_{2}}\right)\right\rceil x_{i j}^{0}+\sum_{j \in J^{0}}\left\lceil\frac{1}{2}\left(\bar{\epsilon}_{j}^{k_{1}}+\bar{\epsilon}_{j}^{k_{2}}\right)\right\rceil y_{j}^{0} .
$$

Now, suppose that the cut induced by $k_{1}$ is stronger than the one induced by $k_{2}$; in this case the resulting zero-half cut $z h$-LS is stronger than the $L$-shaped cut corresponding to $k_{2}$. We use this observation to incorporate zero-half cuts $z h$-LS into the scheme described in Algorithm 1 for separating $L$-shaped cuts as follows: Let $k_{1}$ be the first scenario in $\bar{K}$ that induces an $L$-shaped cut $(l$-LS $)$; afterwards, for each following scenario explored in $\bar{K}$ inducing a violated cut, we generate the corresponding $(l-\mathrm{LS})$ and combine it with the one obtained by $k_{1}$, which yields a stronger violated $z h$-LS). This strategy is justified by the fact that the ordering of the elements in $\bar{K}$ is based on how strong the previously produced cuts have been with respect to the cut violation.

A Matheuristic for Generation of Additional $\boldsymbol{L}$-shaped Cuts We have described how we use the current fractional solution $\left(\tilde{\mathbf{x}}^{0}, \tilde{\mathbf{y}}^{0}\right)$ in order to obtain a collection of valid inequalities of type $(\overline{\mathrm{LS}}),(l$-LS $), \quad z h$-LS $)$ and $(i$-LS $)$. The idea now is to use $\left(\tilde{\mathbf{x}}^{0}, \tilde{\mathbf{y}}^{0}\right)$ in order to heuristically obtain an alternative feasible pair $\left(\hat{\mathbf{x}}^{0}, \hat{\mathbf{y}}^{0}\right)$ and use it to find additional $L$-shaped cuts at the root node of the enumeration tree.

The pair $\left(\hat{\mathbf{x}}^{0}, \hat{\mathbf{y}}^{0}\right)$ is found by a matheuristic that resembles the basic ideas of Local Branching [see 24, 42]. Let $S_{\mathbf{x}^{0}}=\left\{(i, j) \in A^{0} \mid \tilde{x}_{i j}^{0}>\pi\right\}$ and $S_{\mathbf{y}^{0}}=\left\{j \in J^{0} \mid \tilde{y}_{j}^{0}>\pi\right\}$, 
be the sets of first-stage allocation and location decisions, respectively, whose corresponding optimal LP-values are greater than $\pi$, where $\pi$ is a predefined threshold value. If $\left(\tilde{\mathbf{x}}^{0}, \tilde{\mathbf{y}}^{0}\right)$ is integer, sets $S_{\mathbf{x}^{0}}$ and $S_{\mathbf{y}^{0}}$ represent exactly a feasible first-stage solution. Hamming distances of an arbitrary pair $\left(\mathbf{x}^{0}, \mathbf{y}^{0}\right)$ to $\left(\tilde{\mathbf{x}}^{0}, \tilde{\mathbf{y}}^{0}\right)$ can be defined as

$$
\Delta\left(\mathbf{x}^{0}, \tilde{\mathbf{x}}^{0}\right)=\sum_{(i, j) \in S_{\mathbf{x}^{0}}}\left(1-x_{i j}^{0}\right)+\sum_{(i, j) \in A^{0} \backslash S_{\mathbf{x}^{0}}} x_{i j}^{0}
$$

and

$$
\Delta\left(\mathbf{y}^{0}, \tilde{\mathbf{y}}^{0}\right)=\sum_{j \in S_{\mathbf{y}^{0}}}\left(1-y_{j}^{0}\right)+\sum_{j \in J^{0} \backslash S_{\mathbf{y}^{0}}} y_{j}^{0} .
$$

For a given $\left(\tilde{\mathbf{x}}^{0}, \tilde{\mathbf{y}}^{0}\right)$, the alternative solution $\left(\hat{\mathbf{x}}^{0}, \hat{\mathbf{y}}^{0}\right)$ is found as follows. Let $\boldsymbol{\Phi}$ be the set of points $\left(\mathbf{x}^{0}, \mathbf{y}^{0}, \omega\right)$ defined by the cuts of type $(\overline{\mathrm{LS}}),(l$-LS $),(z h$-LS $)$ or $(i$-LS $)$ that have been added to the (MP) so far. The solution $\left(\hat{\mathbf{x}}^{0}, \hat{\mathbf{y}}^{0}\right)$ is found by solving the following LP problem:

$$
\begin{aligned}
\left(\hat{\mathbf{x}}^{0}, \hat{\mathbf{y}}^{0}\right)=\arg \min & \sum_{j \in J^{0}} f_{j}^{0} y_{j}^{0}+\sum_{(i, j) \in A^{0}} c_{i j}^{0} x_{i j}^{0}+\omega \\
\text { s.t. } \quad & \Delta\left(\mathbf{x}^{0}, \tilde{\mathbf{x}}^{0}\right) \leq \kappa_{\mathbf{x}} \\
& \Delta\left(\mathbf{y}^{0}, \tilde{\mathbf{y}}^{0}\right) \leq \kappa_{\mathbf{y}} \\
& \Delta\left(\mathbf{y}^{0}, \tilde{\mathbf{y}}^{0}\right) \geq 1 \\
& \left(\mathbf{x}^{0}, \mathbf{y}^{0}, \omega\right) \in \mathbf{\Phi} \\
& \text { FS.1), } \text { FS.2 and }\left(\mathbf{x}^{0}, \mathbf{y}^{0}\right) \in[0,1]\left|A^{0}\right|+\left|J^{0}\right|,
\end{aligned}
$$

where the constants $\kappa_{\mathbf{x}}$ and $\kappa_{\mathbf{y}}$ of $(\overline{M H .2})$ and (MH.3), respectively, define the neighborhood within which we want to find $\left(\hat{\mathbf{x}}^{0}, \hat{\mathbf{y}}^{0}\right)$. Constraint (MH.4) ensures that the new solution will differ from the original one in at least 1 unit of distance with respect to $\mathbf{y}^{0}$. The later condition is imposed considering that a small change regarding the set of opened facilities is more likely to yield a different (and potentially useful) solution than a change on the allocation decisions.

Once that (MH.1)-(MH.6) is solved, the solution $\left(\hat{\mathbf{x}}^{0}, \hat{\mathbf{y}}^{0}\right)$ is used to obtain cuts of type $(l-\mathrm{LS})$ (or $(z h$-LS $)$ if the feature is enabled) applying the same procedures explained above. Furthermore, we have implemented an iterative process in which problem (MH.1)(MH.6) is solved $M_{h}$ times, such that the neighborhood size is slightly increased in each following iteration. More precisely, at a given iteration $t, \kappa_{\mathbf{x}}$ and $\kappa_{\mathbf{y}}$ are given by:

$$
\kappa_{\mathbf{x}}=\left\lceil(1+t) \times \vartheta \times\left|S_{\mathbf{x}^{0}}\right|\right\rceil \text { and } \kappa_{\mathbf{y}}=\left\lceil(1+t) \times \vartheta \times\left|S_{\mathbf{y}^{0}}\right|\right\rceil
$$

where $\vartheta \in[0,1]$ is a user defined parameter. In our default implementation, parameters $\pi$, $\vartheta$ and $M_{h}$ are set to $0.1,0.75$ and 2 respectively.

It is well-known that the incorporation of constraints such as (MH.2) and (MH.3) usually decreases the practical difficulty of a model [see 24], therefore, finding these additional cuts is computationally inexpensive. 


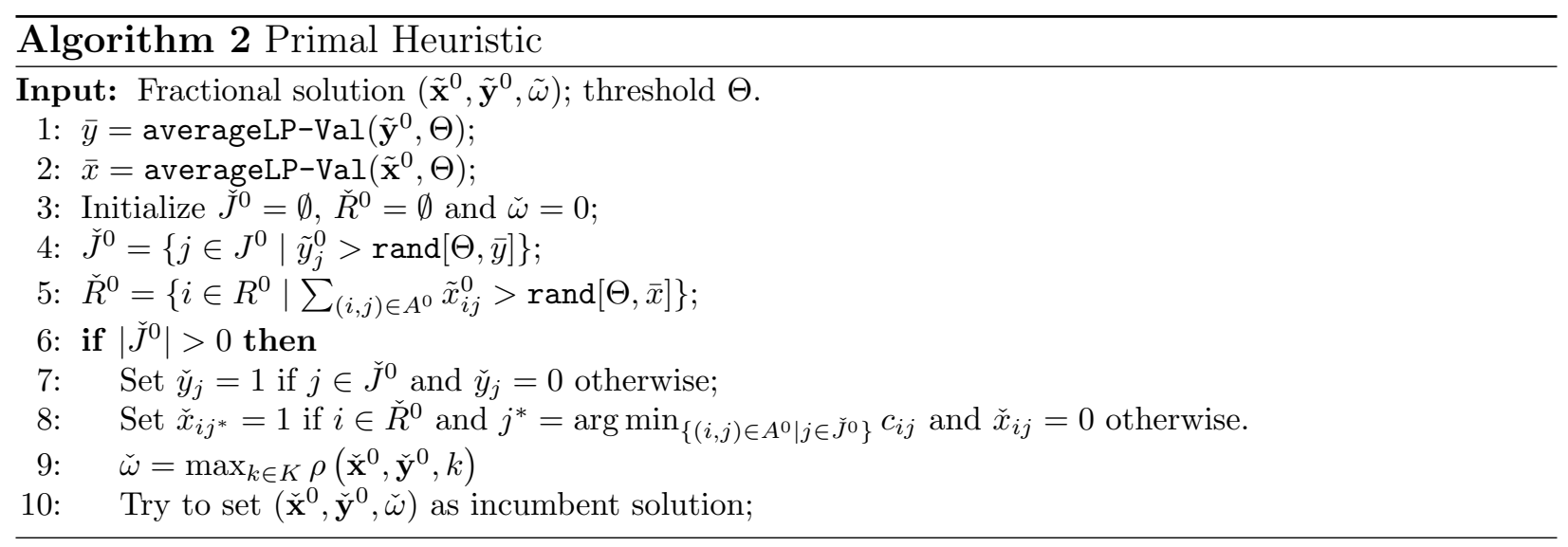

\subsection{Primal Heuristic}

Another component of our algorithm is a primal heuristic that uses the information of the current fractional solution $\left(\tilde{\mathbf{x}}^{0}, \tilde{\mathbf{y}}^{0}\right)$ and attempts to construct a feasible solution $\left(\check{\mathbf{x}}^{0}, \check{\mathbf{y}}^{0}, \breve{\omega}\right)$ that improves the current upper bound. The scheme of the primal heuristic is presented in Algorithm 2.

Function averageLP-Val $\left(\tilde{\mathbf{y}}^{0}, \Theta\right)$ (see line 1), is given by

$$
\frac{\sum_{j \in J^{0}: \tilde{y}_{j}>\Theta} \tilde{y}_{j}^{0}}{\left|J^{0}: \tilde{y}_{j}^{0}>\Theta\right|} ;
$$

which means that $\bar{y}$ is computed using only those elements whose LP-values are larger than $\Theta$, where $\Theta$ is a predefined threshold value. The value $\bar{x}$ is computed similarly (see line 2).

A key element of the proposed heuristic is given in lines 4 and 5 . set $\check{J}^{0}$ (resp. $\check{R}^{0}$ ) is built by adding an element $j$ (resp. $i$ ) if $\tilde{y}_{j}^{0}\left(\operatorname{resp} . \sum_{(i, j) \in A^{0}} \tilde{x}_{i j}^{0}\right)$ is greater than a value, uniformly randomly generated in the interval $[\Theta, \bar{y}]$ (resp. $[\Theta, \bar{x}]$ ). Thanks to the use of average LP-values $\bar{x}$ and $\bar{y}$, important information about the solution topology is transferred from the current LP solution to the heuristic solution. On the other hand, the use of random thresholds (lines 4 and 5 p provides diversification to the heuristic and helps in escaping local optima. The feasible first-stage solution $\left(\check{\mathbf{x}}^{0}, \check{\mathbf{y}}^{0}\right)$ is computed in lines 7 and 8 by means of a very simple greedy heuristic. The heuristic value of $\check{\omega}$ is found in line 9. Although $|K|$ ILP problems (R.1)-(R.6) have to be solved they are not solved to optimality but until a gap of less than $1 \%$ is reached (which typically takes at most a few seconds). The default value of $\Theta$ was set to 0.01 .

\subsection{Auxiliary Variables and Branching Priorities}

Looking more carefully at the objective function of a $k$-th subproblem, one easily observes that for each customer $i \in R$, its assignment variables are grouped together into binary decisions: (i) the customer is served in the first stage $\left(\sum_{(i, j) \in A^{0}} x_{i j}^{0}\right)$, and (ii) the customer is served in the first stage by a wrong facility $\left(\sum_{(i, j) \in A^{0} \backslash A^{k}} x_{i j}^{0}\right)$. This motivates us to introduce additional binary decision variables and impose a new non-standard branching on them. More precisely, we introduce auxiliary binary variables $\mathbf{q}, \mathbf{s} \in\{0,1\}^{\left|R^{k}\right|}$, for all $k \in K$, as 
follows:

$$
\begin{aligned}
q_{i}^{k} & =\sum_{(i, j) \in A^{0}} x_{i j}^{0}, \quad \forall i \in R^{k}, \forall k \in K \\
s_{i}^{k} & =\sum_{(i, j) \in A^{0} \backslash A^{k}} x_{i j}^{0}, \quad \forall i \in R^{k}, \forall k \in K .
\end{aligned}
$$

These auxiliary variables play two important roles in our algorithmic framework. First, they are useful in the efficient construction of the LP (and ILP) SPs. The right-hand-side of (R.2) and (R.3) can be fixed for each $i \in R^{k}$ without the need of any extra loop to sum up the values of the first-stage solution $\tilde{x}^{0}$. Second, and more important, these auxiliary variables are used to guide the branching in a more effective way by imposing higher branching priorities on them. Clearly, fixing to 0 or to 1 one of these variables immediately fixes the value of other variables. For instance if $q_{i}^{k}=1$ and $s_{i}^{k}=0$ for a given $i \in R^{k}$ (customer $i \in R^{k}$ has been allocated in the first-stage to a facility through a link that is available in scenario $k$ in the second stage), then $x_{i j}^{k}=z_{i j}^{k}=0 \forall(i, j) \in A^{k}$. Otherwise, if $q_{i}^{k}=0$ (customer $i \in R^{k}$ has not been allocated in the first-stage to any facility), then $s_{i}^{k}=0, \sum_{i \in R^{k}} x_{i j}^{k}=1$ and $z_{i j}^{k}=0$ $\forall(i, j) \in A^{k}$. Other combinations can be analyzed straightforwardly.

Adding these variables and constraints (5)-(6) does not modify the polyhedral characterization of (1)-(2), so the computational effort does not intrinsically change by including them.

\section{Computational Results}

In this section we first introduce two sets of benchmark instances that resemble application of facility location in transportation networks and in the disaster management, respectively. We use these instances (i) to analyze the properties of the obtained solutions and their dependence on the cost structure, (ii) for showing the advantages of the recoverable robustness, and (iii) for assessing the performance of the proposed branch-and-cut algorithm. Finally, we also compare the performance of the proposed algorithm with the performance of CPLEX when solving formulation (1)-(2) directly (i.e., as a compact model).

All the experiments were performed on an Intel Core ${ }^{\mathrm{TM}}$ i7 (4702QM) $2.2 \mathrm{GHz}$ machine (8 cores) with 16 GB RAM. The branch-and-cut was implemented using CPLEX ${ }^{\mathrm{TM}} 12.5$ and Concert Technology framework. When testing our branch-and-cut all CPLEX parameters were set to their default values, except the following ones: (i) All cuts were turned off, (ii) heuristics were turned off, (iii) preprocessing was turned off, (iv) the time limit was set to 600 seconds. Besides, higher branching priorities were given to $\mathbf{y}^{0}$ and to the auxiliary variables $\mathbf{q}$ and $\mathbf{s}$ as described in $\$ 3.3$.

We have turned some CPLEX features off (only when running our algorithm) in order to make a fair assessment of the performance of the techniques described in $\$ 3$.

\subsection{Benchmark Instances}

We consider two classes of instances, that we refer to as Trans and Dis. Instances of the first class are intended to resemble real transportation networks in which the transportation

costs depend on both the distance to be covered and the amount of commodities to be 
transported, and where the set-up cost of facilities strongly depends on the demographic characteristics of the corresponding (urban) area. Dis instances approximate situations such as humanitarian relief in natural disasters in which some transportation links are interdicted, i.e., they are damaged so that the transportation time can be severely increased. We assume that if a given city $i \in R^{k}$ requires to be served but each path from any $j \in J^{k}$ to $i$ contains at least one interdicted link, then the city is still assisted although at a very high response time. Besides, set-up costs $f_{j}^{0}$ are such that one might favor to install facilities in cities where the average distance to all the potential customers is relatively small.

Trans Instances In this class of instances we consider three groups: US, Germany and ND-I. In groups US and Germany we consider the geographical coordinates and updated data of population of the 500 most populated cities in each country [see 47]. In group ND-I we consider random instances with up to 500 nodes randomly located in a unit square and population being an integer number taken uniformly at random from the interval $[1 \times$ $\left.10^{4}, 2.5 \times 10^{6}\right]$. We denote by $d_{i j}$ the Euclidean distance between cities $i$ and $j$, and by pop $_{i}$ the population size of city $i$.

Given the coordinates and the population size associated with each node, an instance of the RRUFL is then generated as follows:

(i) take the first $n$ cities in terms of population;

(ii) define $R^{0}$ by randomly selecting $50 \%$ of the cities;

(iii) for $k \in K$ define $R^{k}$ by randomly taking $\left|R^{0}\right| \times \operatorname{rand}[0.4,0.6]$ cities from $R^{0}$;

(iv) for $k \in K$ define $J^{k}$ by randomly taking $\left(n-\left|R^{k}\right|\right) \times \operatorname{rand}[0.2,0.3]$ cities from $1, \ldots, n$ $\left(J^{0}=\cup_{k \in K} J^{k}\right)$;

(v) for $k \in K$ define $A^{k}=R^{k} \times J^{k}\left(A^{0}=R^{0} \times J^{0}\right)$;

(vi) first- and second-stage transportation/allocation costs are defined as $c_{i j}^{0}=d_{i j} \times \frac{1}{2}\left(\right.$ pop $_{i}+$ $\left.\operatorname{pop}_{j}\right) \times \varphi, c_{i j}^{k}=\left(1+\sigma_{1}\right) \times c_{i j}^{0}$ and $r_{i j}^{k}=\left(1+\sigma_{2}\right) \times c_{i j}^{0}$ for $k \in K$;

(vii) first- and second-stage set-up costs and penalties are defined as $f_{j}^{0}=\rho \times$ pop $_{j}, f_{j}^{k}=$ $\left(1+\sigma_{3}\right) \times f_{j}^{0}$ and $p_{j}^{k}=\left(1+\sigma_{4}\right) \times f_{j}^{0}$ for $k \in K$.

All coefficients are finally rounded to their nearest integer values.

Parameter $\varphi$ is given in $\$$ per unit of distance per unit of demand, so the allocation costs are purely expressed in $\$$; parameter $\rho$ is given in $\$$ per inhabitant (so the larger a city is, the more expensive the set-up of a facility is); parameters $\sigma_{1}, \sigma_{2}, \sigma_{3}$ and $\sigma_{4}$ are [0,1] factors representing the increase of the allocation and set-up costs in the second stage.

The possibility that a location (and the corresponding facility) becomes unavailable in the second stage, stems from practical applications. For example, from a long term perspective, changes in the environmental legislation might force to stop the construction of a facility, or it might impose environmental mitigation costs that turn the project infeasible. Similarly, from a short term perspective, a facility might become unavailable due to a labor strike. Our purpose is to present a model of uncertainty that covers different situations as these two. To this end, a subset of facilities, about 40-60\% of them (depending on the scenario, see 


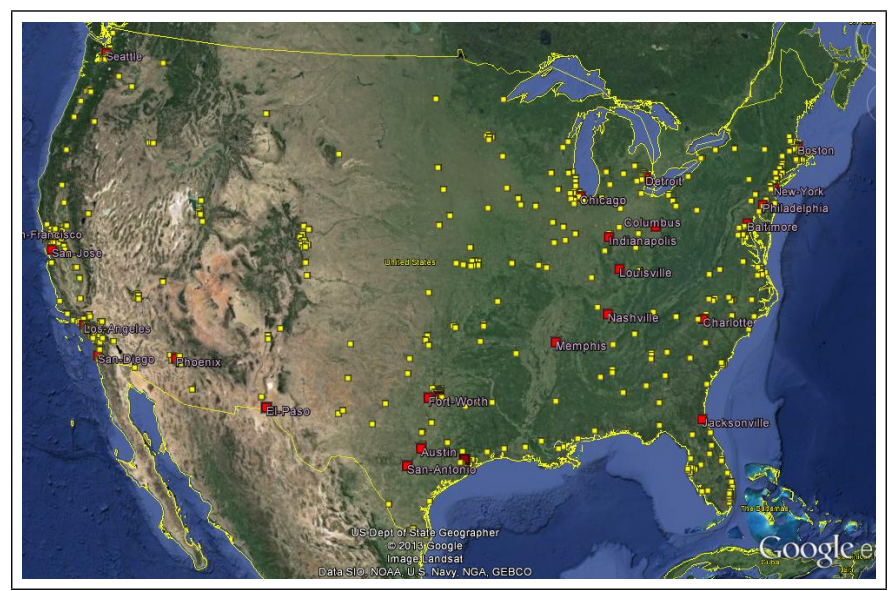

(a) Trans-US

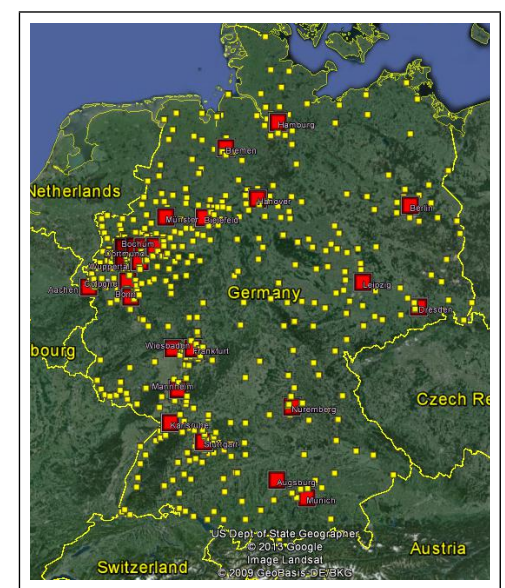

(b) Trans-Germany

Figure 2: Representation of Trans Instances.

(iii)) are subject to failure in the second stage. Notice that, by construction, not necessarily all facilities are subject to failure, but only those that become unavailable in at least one scenario (i.e., $J^{0} \backslash \cap_{k} J^{k}$ ). A similar scheme applies for the sets of customers $R^{k}$.

As can be seen from (v), for this set of instances we do not consider disruptions in the transportation network, i.e., for a given $k \in K$, all customers in $R^{k}$ can be reached from every facility in $J^{k}$ (with higher single-stage costs, though).

Figures 2(a) and 2(b) show the graphical representation of the 500 cities used in groups US and Germany respectively (the name of the first 25 cities are provided). For $n=500$, each scenario resembles a UFL instance with $\approx 125$ customers and $\approx 100$ locations (the sets $J^{k}$ and $R^{k}$ may intersect).

To create a large set of benchmark instances, we use the following parameter settings: $n \in$ $\{100,250,500\}, \varphi \in\left\{10^{-5}, 10^{-4}, 10^{-3}, 10^{-2}\right\}, \rho \in\{0.001,0.01,0.1,1.0\} \sigma_{1}, \sigma_{2} \in\{0.05,0.50\}$, and $\sigma_{3}, \sigma_{4} \in\{0.10,1.0\}$. In our computations we consider up to 75 scenarios which are created in advance. By doing this, when dealing with instances with 25 scenarios, we simply use the first 25 scenarios out of those 75 . The same applies for 50 scenarios. The scenarios are identical for the different values of all other parameters. By proceeding in this way, it is easier to measure the impact of considering a larger number of scenarios. For a given group (US, Germany, or ND-I) there are $3 \times 4 \times 4 \times 2 \times 2 \times 2 \times 2 \times 3=2304$ instances to be solved.

Dis Instances In this class of instances we consider three groups: Bangladesh, Philippines and ND-II. In group Bangladesh (resp. Philippines) we consider the geographical coordinates and updated data of population of the 128 (resp. 100) most populated cities in each case [see 47]; in group ND-II we consider random instances with 100 nodes randomly located in a unit square and the size of the population is taken uniformly at random from $\left[1 \times 10^{4}, 2.5 \times 10^{6}\right]$. In the case of groups Bangladesh and Philippines we use pairwise Euclidean distances between selected cities and embed them in a network $N=(V, A)$, with $V$ being the set of $n$ cities and $A$ the allocation links $(n=128$ for group Bangladesh and $n=100$ for group Philippines). For the case of the group ND-II, the network $N=(V, A)$ is obtained such that a link is established between two cities $i$ and $j$ if the Euclidean distance 


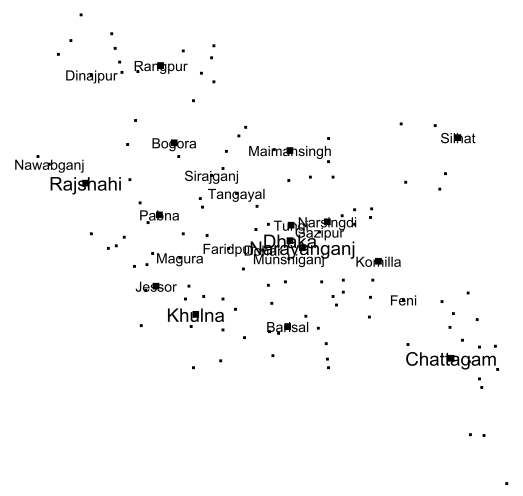

(a) Nodes of the input network

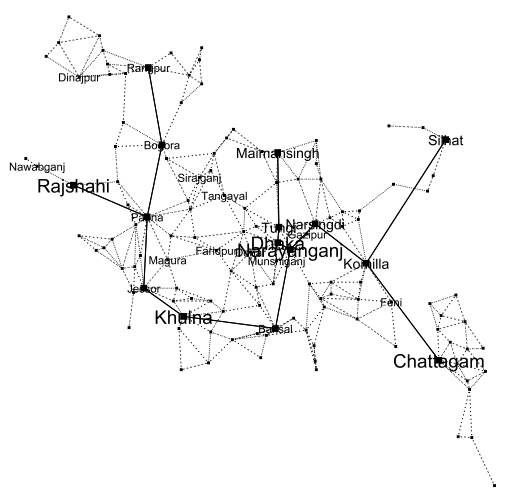

(b) $N=(V, A)$ after adding links

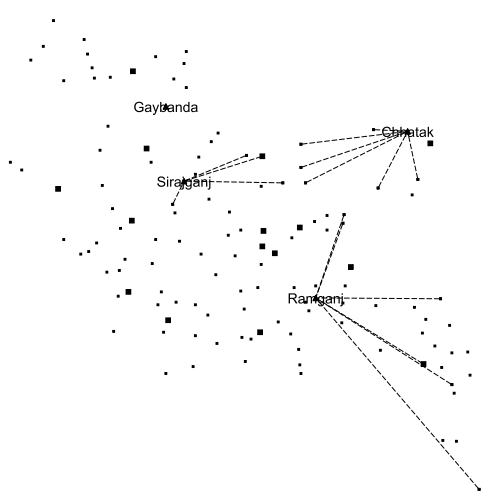

(c) Example of a 1st-stage solution

Figure 3: Construction process of Dis Instances and an example of a first-stage solution. Bangladesh Instances.

is smaller than or equal to $\alpha / \sqrt{n}$ ( $\alpha$ is an input parameter fixed to 1.6 in our computations). Figure 3(a) shows the location of the 128 cities for the Bangladesh group of instances, Figure 3(b) illustrates the embedded network $N=(V, A)$ of the same group, and Figure 3(c) shows an example of a first-stage solution.

With the information of each group, Bangladesh, Philippines or ND-II, an instance of the RRUFL is generated as follows:

(i) define $R^{0}$ by randomly selecting $t \%$ of the cities, with $t \in\{25,50,75\}$;

(ii) for $k \in K$ define $R^{k}$ by randomly taking $\left|R^{0}\right| \times \operatorname{rand}[0.4,0.6]$ cities from $R^{0}$;

(iii) for $k \in K$ define $J^{k}$ by randomly taking $\left(n-\left|R^{k}\right|\right) \times \operatorname{rand}[0.08,0.12]$ cities from $1, \ldots, n$ $\left(J^{0}=\cup_{k \in K} J^{k}\right)$;

(iv) first-stage allocation $\operatorname{costs} c_{i j}^{0}$ are equal to the shortest path cost between $i$ and $j$ in $N=(V, A)$ using Euclidean distances $d_{u v}, \forall\{u, v\} \in A$;

(v) for the second-stage allocation costs we consider random link interdiction, that is: let $I^{k}$ be a set of $f \times|A| \times \operatorname{rand}[0.8,1.2]$ links randomly chosen from $A$. Then $d_{u v}^{k}=d_{u v}$, for all $\{u, v\} \in A \backslash I^{k}$, and $d_{u v}^{k}=100 \times d_{u v}$, for all $\{u, v\} \in I^{k}$, so $c_{i j}^{k}$ is equal to the cost of the shortest path between $i$ and $j$ with edge costs given by $\mathbf{d}^{k}$. Reallocation cost $r_{i j}^{k}$ is $1.5 \times d_{i j}^{k}$, for $k \in K$;

(vi) first-stage set-up costs are given by $f_{j}^{0}=\sum_{i \in R^{0}} c_{i j}^{0} /\left|R^{0}\right|$, and second-stage set-up and penalty costs are given by $f_{j}^{k}=\left(1+\sigma_{3}\right) \times f_{j}^{0}$ and $p_{j}^{k}=\left(1+\sigma_{4}\right) \times f_{j}^{0}$, for $k \in K$.

The remaining parameters are $f \in\{0,0.10,0.25,0.50\}, \sigma_{3}=\{0.00,1.00\}$ and $\sigma_{4}=$ $\{0.10,1.0,4.0\}$. All possible parameter settings, in combination with $k \in\{25,50,75\}$ imply that there are $3 \times 4 \times 2 \times 3 \times 3=216$ instances to be solved for each fixed value of $n$ within each group. 


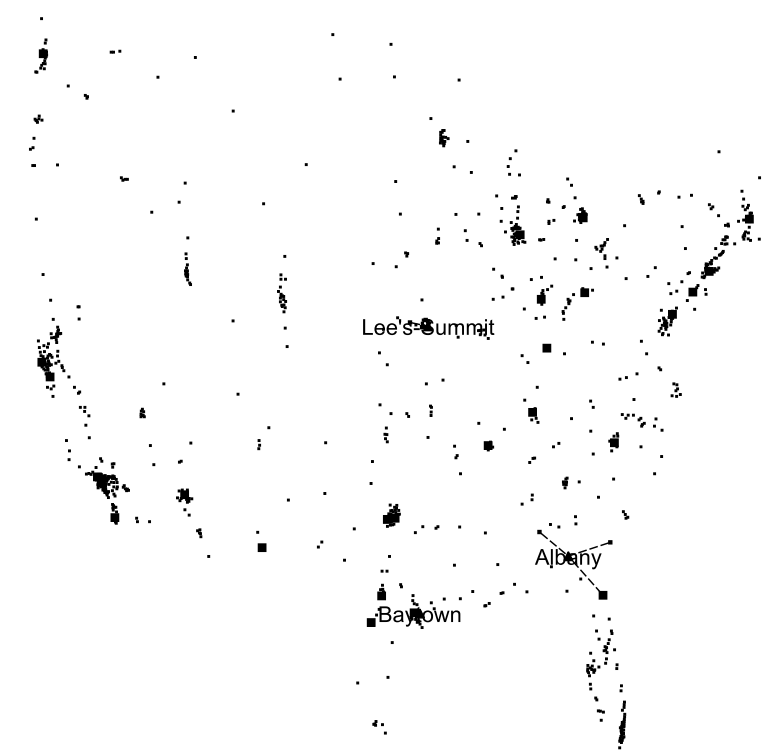

(a) (0.1,1.0), $\left|\mathbf{y}^{0}\right|=3,\left|\mathbf{x}^{0}\right|=8$

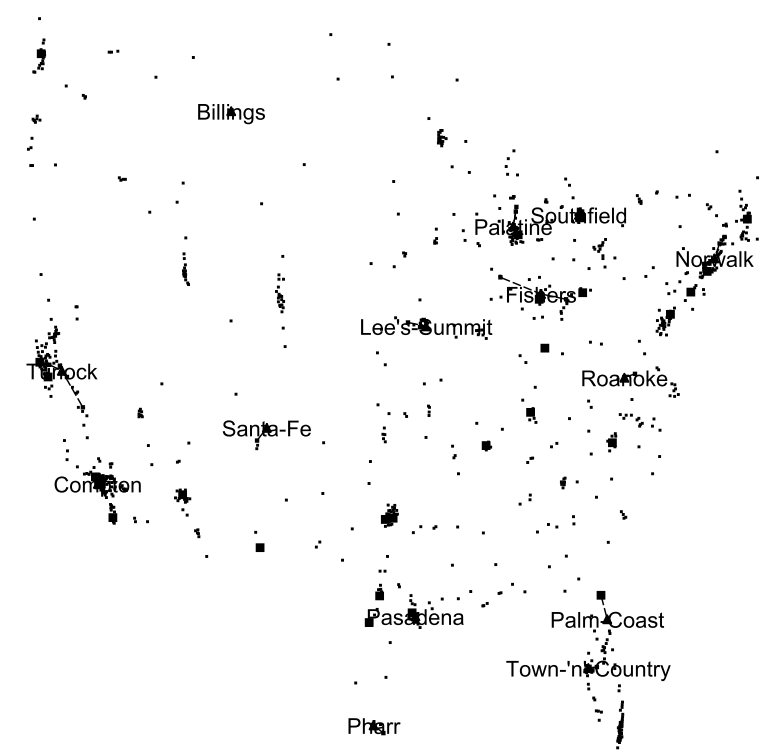

(b) (1.0,0.1), $\left|\mathbf{y}^{0}\right|=14,\left|\mathbf{x}^{0}\right|=54$

Figure 4: Solutions considering different combinations of $\left(\sigma_{3}, \sigma_{4}\right)$ (Instances US, $n=500, \varphi=0.001, \rho=0.1$ $\sigma_{1}=0.5, \sigma_{2}=0.05$ and $\left.|K|=25\right)$

\subsection{Trans Instances: Solutions, Robustness and Recoverability}

Influence of the Cost Structure The characteristics of a robust first-stage solution and the corresponding recovery actions depend not only on the scenario structure but also on the cost structure. If, for example, for a given instance the second-stage costs are very high with respect to the first-stage costs then the solutions of the RRUFL will tend to have more facilities and assignments defined in the first stage. Likewise, if the second-stage set-up costs are much higher than the penalty costs $\left(f_{j}^{k}>>p_{j}^{k}\right)$, we would expect that more facilities will be opened in the first-stage (and eventually more assignments) than if $f_{j}^{k} \leq p_{j}^{k}$, where the cost of setting-up a facility in the second stage is cheaper than the penalty for a facility placed at a non-available location.

In Figure 4 we show the later case by comparing two solutions of an instance of group US. For the first one (Figure 4(a)), the penalties are $\approx 81 \%$ more expensive than the second-stage set-up costs, while for the second one (Figure 4(b)), the penalties are $45 \%$ cheaper. We can see how changing the relation between $f_{j}^{k}$ and $p_{j}^{k}$ leads to very different solutions: while in the first case 3 facilities are opened in the first stage and 8 customers are allocated to them, in the second case 14 facilities are opened in the first stage and 54 customers are allocated.

The relation between parameters $\varphi$ (\$ per unit of distance per inhabitant) and $\rho$ (\$ per inhabitant), also influences the solution structure. Assume that we are given an instance with $\varphi<\rho$ (set-up costs are higher than the allocation costs) and another instance with $\varphi>\rho$ (allocation costs are higher than the set-up costs). We would expect that the solution of the second instance will be comprised by a larger first-stage component compared to the solution of the first instance. Figure 5 depicts this by comparing the solution obtained for $\varphi=0.0001$ and $\rho=0.01$ (Figure 5(a)) with the one obtained for $\varphi=0.01$ and $\rho=0.001$ (Figure 5(b)]. In the first case, only a single facility is open in the first stage and 5 allocations are defined, 


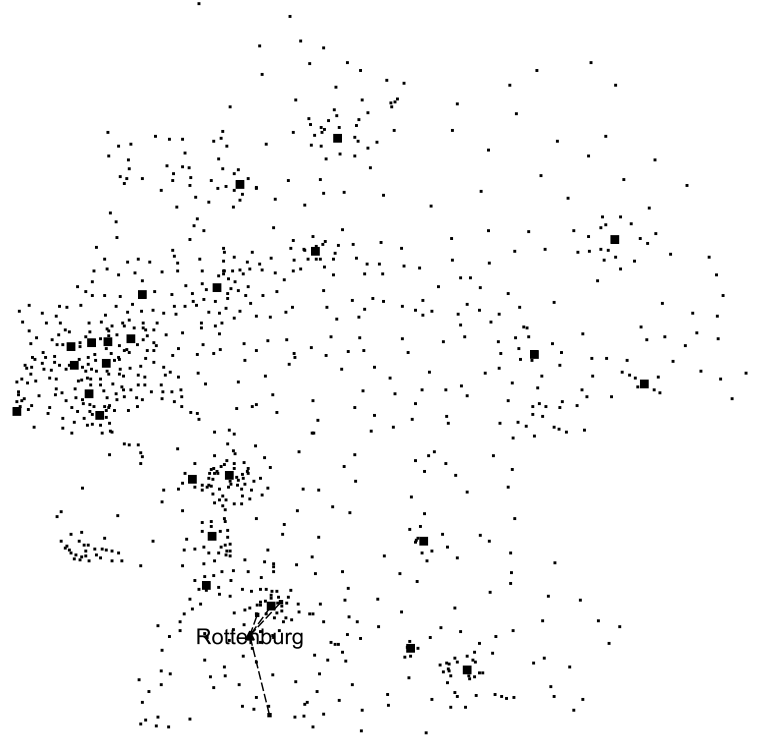

(a) $(0.0001,0.01),\left|\mathbf{y}^{0}\right|=1,\left|\mathbf{x}^{0}\right|=5$

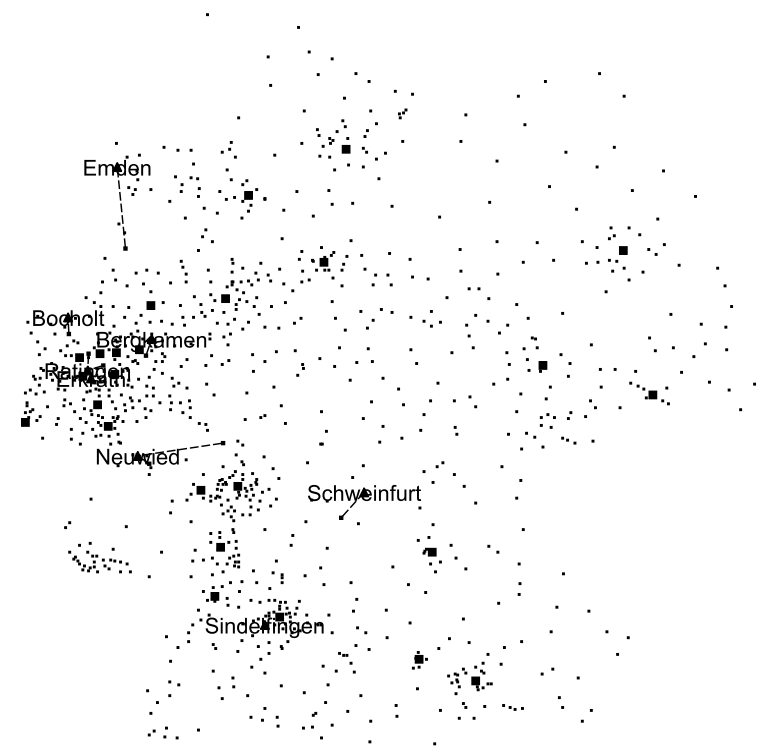

(b) $(0.01,0.001),\left|\mathbf{y}^{0}\right|=8,\left|\mathbf{x}^{0}\right|=12$

Figure 5: Solutions considering different combinations of $(\varphi, \rho)$ (Instances Ger, $n=250, \sigma_{1}=0.5, \sigma_{2}=0.5$ $\sigma_{3}=0.1, \sigma_{4}=1.0$ and $\left.|K|=25\right)$

while in the second case 8 facilities are installed and 12 allocations are established in the first stage. This effect is quite intuitive considering that the second stage costs are proportional to the first stage costs for these instances: it is better to open facilities in the same place where the demand is located, i.e., in a subset of $R^{0} \cap J^{0}$, in order to avoid high allocation expenses $(\varphi>\rho)$.

A more extensive analysis on the influence of the second-stage cost parameters $(\varphi, \rho)$ and $\left(\sigma_{1}, \sigma_{2}, \sigma_{3}, \sigma_{4}\right)$ on the performance of the algorithm and on the the solutions characteristics is now presented. In Figure 6(a) we show the box-plots of the attained gaps for all the combinations of $(\varphi, \rho)$ when solving Germany group with $n=250$. Each box-plot contains information about 48 instances. The maximum and attained gaps are marked with a bold circle and an asterisk, respectively, and the number of instances solved to optimality is displayed under each box-plot. Recall that $\varphi$ is a factor expressed in $\$$ per unit of distance per unit of demand, and $\rho$ is expressed in $\$$ per inhabitant. We can observe the following: (i) The problem becomes easier (more instances can be solved to optimality) when $\varphi$ is considerably smaller than $\rho\left(10^{3}-10^{5}\right.$ times smaller $)$, that is, for those instance where the set-up costs are considerably higher than the operating costs (transportation). (ii) When $\rho<\varphi$ we have that the transportation costs are larger than the set-up costs; in these cases the attained gaps are relatively small. (iii) The problems become harder when $\frac{\varphi}{\rho} \geq 10^{-2}$. These three behaviors can be explained by the fact that in the easier first two cases there is not as much symmetry in the cost structure between opening and transportation costs as in in the third case (where the opening and transportation costs are of the same magnitude).

In Figure 6(b) we show the box-plots of the attained gaps for the 16 combinations of $\left(\sigma_{1}, \sigma_{2}, \sigma_{3}, \sigma_{4}\right)$. Average and maximum gaps are marked with bold circles and asterisk as before, and under each box-plots we provide the average value of the number of facilities 


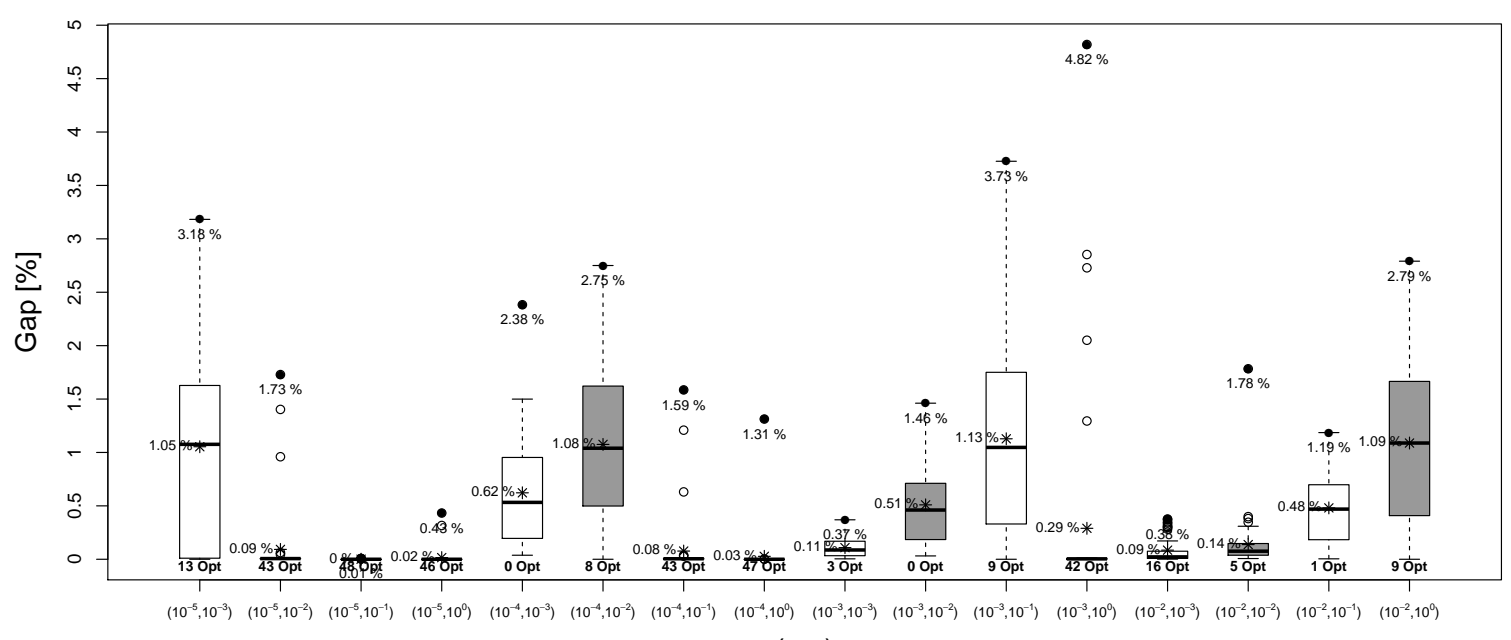

$(\varphi, \rho)$

(a) Box-plots of attained Gap (\%) vs. $(\varphi, \rho)$

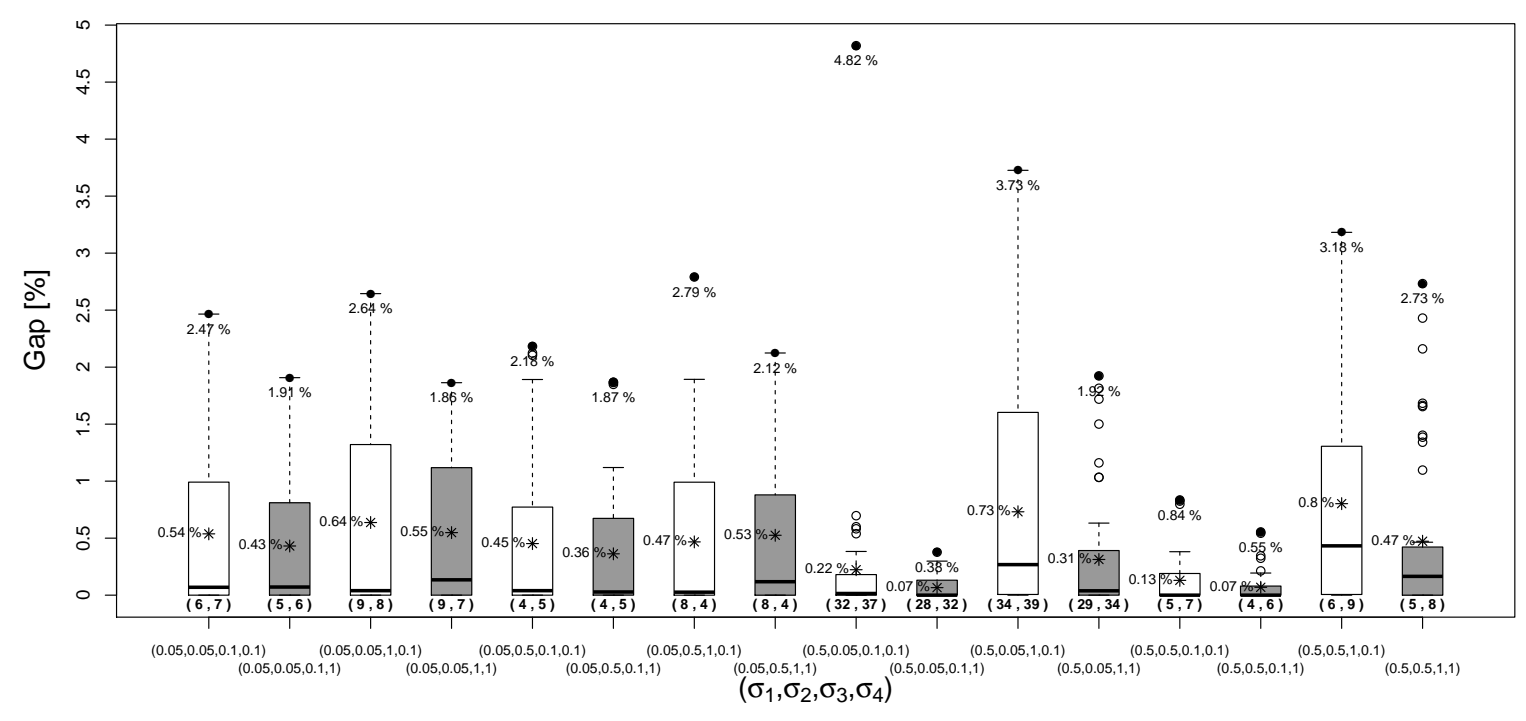

(b) Box-plots of attained Gap (\%) vs. $\left(\sigma_{1}, \sigma_{2}, \sigma_{3}, \sigma_{4}\right)$

Figure 6: Influence of cost parameters $(\varphi, \rho)$ and $\left(\sigma_{1}, \sigma_{2}, \sigma_{3}, \sigma_{4}\right)$ on the algorithmic performance and the solution structure (Group Germany, $n=250$ )

open in the first stage and the number of first-stage decisions (opened facilities and defined allocations). From this graphic, one can highlight the following observations: (i) The largest first-stage components (as well as high gaps) are obtained when the factor of the re-allocation cost $\sigma_{2}$ is 0.05 and, especially, when $\sigma_{1}=0.5$ (the increasing factor of the second-stage allocation costs). (ii) The algorithmic performance is considerably more stable (but not better on the average) when $\sigma_{1}$ is 0.05 than when it is 0.5 . (iii) The algorithm behaves better when the penalty factor $\sigma_{4}$ is 0.1 than when it is 1.0 (the difference is more clear 
when $\left.\sigma_{1}=0.5\right)$. These outcomes can be explained as follows. When the second-stage allocation costs are expensive (50\% higher the first-stage value), but the re-allocation costs are cheap (only $5 \%$ higher), then an optimal or nearly optimal first-stage solution will tend to consist of several allocations which, therefore, implies that several facilities have to opened in the first-stage. On the other hand, if both costs are expensive $\left(\sigma_{1}=\sigma_{2}=0.5\right)$, then having a large first-stage component does not pay off. Having expensive second-stage allocation costs $\left(\sigma_{1}=0.5\right)$ implies that the $\mathbf{x}^{k}$ variables will likely be equal to 0 (regardless of $k$ ); this immediately reduces the average computational effort of the separation problem. At the same time, this implies that a good first-stage policy is required for having a globally good solution. However, such a first-stage solution might be hard to find quickly, which explains the large dispersion of gaps observed when $\sigma_{1}=0.5$. Likewise, if the penalty paid for having a first-stage facility in a non-available location is expensive $\left(\sigma_{4}=1.0\right)$, then the first-stage solutions will tend to consist of as few facilities as possible (so the total second-stage penalty for the misplaced facilities is as small as possible); again, the need of a good first-stage policy (at least better than when $\sigma_{4}=0.1$ ) explains why the problem becomes harder, especially when a greater value of $\sigma_{1}$ pushes towards solutions with more facilities opened in the first stage.

The Gain of Recovery A more accurate measure of the benefits of the recovery can be calculated by comparing the solutions obtained for the RRUFL with those obtained for the RUFL presented in $\$ 2.1$. Recall that the RUFL model is such that facilities can only be opened in the first stage, whereas allocations can only be established in the second stage. Hence, no recovery actions (in terms of setting-up new facilities or re-allocating customers) are allowed. To illustrate the benefits of the recovery, we now define a measure that we will refer to as the Gain of Recovery $(G o R)$. GoR is defined as the relative gain in terms of cost when using the solution produced by our recoverable robust approach instead of the one produced by the approach without recovery (the RUFL, in our case).

In Table 1 we report on statistics regarding the GoR. Columns $G o R\left(O P T_{\mathcal{R} \mathcal{R}}\right)$ correspond to statistics of the $G o R$ defined as $\operatorname{GoR}\left(O P T_{\mathcal{R} \mathcal{R}}\right)=\frac{O P T_{R}-O P T_{\mathcal{R} R}}{O P T_{R}} \times 100 \%$, where $O P T_{R}$ is the objective function value produced by the RUFL. Columns $G o R\left(O P T_{\omega}\right)$ correspond to statistics of the $G o R$ defined as $\operatorname{GoR}\left(O P T_{\omega}\right)=\frac{\omega_{R}-\omega}{\omega_{R}} \times 100 \%$, where $\omega_{R}$ is the worst-case second stage cost for the RUFL. The obtained values emphasize the practical benefits of recoverable robustness in cases in which recovery is possible; both, the costs of the complete policy (first- and second-stage solutions) and the worst-case second stage solutions are on average 25-40\% cheaper (and the difference can scale above 90\%). These results clearly justify the benefits of the recovery in the second stage, when compared to a less flexible decision making policy.

The Effort for Robustness and the Price of Robustness The more scenarios (possible data realizations) we take into account, the more robust the first-stage solution is expected to be. Nonetheless, this additional robustness is obtained at the expenses of (i) an increase of the difficulty of the problem, since a larger search space must be considered, and (ii) an increase of the total solution cost, $O P T_{\mathcal{R} \mathcal{R}}$, because more facilities have to be opened and more allocations have to be established in the first stage or because a new worst-case scenario induces a higher robust recovery cost (i.e., $\omega$ increases). The first of these effects has been coined as the Effort for Robustness in [5] ; the second effect is similar to what is 


\begin{tabular}{|c|c|c|c|c|c|c|c|}
\hline \multirow{2}{*}{ Group } & \multirow[b]{2}{*}{$|K|$} & \multicolumn{3}{|c|}{$\operatorname{GoR}\left(O P T_{\mathcal{R} \mathcal{R}}\right)$} & \multicolumn{3}{|c|}{$\operatorname{GoR}(\omega)$} \\
\hline & & Median & Ave. & $\operatorname{Max}$ & Median & Ave. & $\operatorname{Max}$ \\
\hline \multirow{3}{*}{ US } & 25 & 34.73 & 36.28 & 89.07 & 31.55 & 33.96 & 82.87 \\
\hline & 50 & 38.64 & 39.30 & 91.94 & 35.52 & 37.31 & 87.46 \\
\hline & 75 & 41.34 & 40.81 & 93.50 & 34.61 & 36.29 & 89.95 \\
\hline \multirow{3}{*}{ Ger } & 25 & 29.17 & 32.73 & 90.01 & 24.91 & 26.39 & 84.37 \\
\hline & 50 & 29.44 & 34.39 & 92.65 & 26.66 & 28.31 & 88.61 \\
\hline & 75 & 32.52 & 37.26 & 93.90 & 28.40 & 32.05 & 90.62 \\
\hline \multirow{3}{*}{ ND-I } & 25 & 24.69 & 25.31 & 79.84 & 29.41 & 30.24 & 70.47 \\
\hline & 50 & 23.47 & 25.75 & 83.49 & 22.57 & 26.18 & 75.79 \\
\hline & 75 & 24.12 & 26.79 & 85.22 & 22.76 & 27.33 & 78.32 \\
\hline
\end{tabular}

Table 1: Statistics of two measures of the Gain of Recovery for different values of $n$ and $|K|$ (Groups US, Germany and ND-I with $n=100$ )

called the Price of Robustness in [10].

To illustrate these effects, in Table 2 we report average values of the results obtained for groups US, Germany and ND-I for varying number of nodes and scenarios (columns Group, $n$ and $|K|$, respectively). The presented values are related to the solution characteristics and to the algorithmic performance. Each row corresponds to the results of 256 instances. Column Time [s] reports the average running times expressed in seconds; column Gap (\%) shows the average gaps attained within the time limit; the average number of facilities opened in the first stage is reported in column $\left|\mathbf{y}^{0}\right|$ and the average number of first-stage allocations is given in column $\left|\mathbf{x}^{0}\right|$; in columns $\triangle O P T \%$ and $\Delta \omega \%$ we report the average relative increase in the value of $O P T_{\mathcal{R} \mathcal{R}}$, resp. $\omega$, when considering 50 and 75 scenarios with respect to the value obtained for 25 scenarios. In column \#Opt the number of instances that were solved to optimality (out of 256) is shown.

The Effort for Robustness is clearly illustrated by the worsening of the algorithmic performance when increasing the number of scenarios: (i) the running times increase (cf. column Time [s]); (ii) the attained gaps increase (cf. column Gap (\%)); and, hence, the number of solutions solved to optimality (cf. column \#Opt) decreases.

The Price of Robustness is demonstrated in columns $\triangle O P T \%$ and $\Delta \omega \%$, where one can see that, without exception, the average values of the solution cost and the robust recovery cost increase when increasing $|K|$ from 25 to 50 and from 25 to 75 . Recall that in our model, decision maker needs to implement only the firt stage solution. In columns $\left|\mathbf{y}^{0}\right|$ and $\left|\mathbf{x}^{0}\right|$ one can see that the size of the first-stage solution is more or less constant for a given $n$, regardless of the value of $|K|$. Hence, increasing the number of scenarios does not produce a measurable effect on the size of the first-stage solution but only on the structure of the second-stage recovery actions (which induces a higher value of $\omega$ ). Similarly, the major economical impact of increasing the number of scenarios is on the costs of the recovery actions. We observe that in all cases (except for two entries of the group Germany) the value of $\triangle O P T \%$ is smaller than the value of $\Delta \omega \%$. This means that the obtained first-stage solutions are such that they allow to reduce the impact of a higher robust recovery cost by balancing robustness and recoverability. The two entries in which the average value of $\triangle O P T \%$ is greater than 


\begin{tabular}{|c|c|c|c|c|c|c|c|c|c|c|c|c|c|}
\hline Group & $n$ & $|K|$ & Time [s] & Gap (\%) & $\left|\mathbf{y}^{0}\right|$ & $\left|\mathbf{x}^{0}\right|$ & $\triangle O P T \%$ & $\Delta \omega \%$ & $\# l-\mathrm{LS}$ & $\#(l-\mathrm{LS})_{\mathrm{MH}}$ & $\# i$-LS & $\# \mathrm{BBN}$ & $\# \mathrm{Opt}$ \\
\hline \multirow{9}{*}{ US } & 100 & 25 & 44.94 & 0.00 & 5 & 7 & 0.00 & 0.00 & 54 & 4 & $\overline{0}$ & 342 & 251 \\
\hline & & 50 & 72.09 & 0.01 & 5 & 6 & 0.12 & 0.64 & 79 & 4 & 1 & 284 & 252 \\
\hline & & 75 & 86.56 & 0.01 & 5 & 6 & 1.11 & 5.23 & 96 & 3 & 0 & 219 & 250 \\
\hline & 250 & 25 & 243.26 & 0.18 & 11 & 14 & 0.00 & 0.00 & 105 & 9 & 0 & 183 & 175 \\
\hline & & 50 & 229.45 & 0.06 & 11 & 11 & 2.89 & 5.61 & 89 & 3 & 0 & 125 & 197 \\
\hline & & 75 & 285.92 & 0.07 & 11 & 11 & 3.50 & 6.68 & 99 & 2 & 0 & 84 & 172 \\
\hline & 500 & 25 & 458.06 & 1.10 & 18 & 25 & 0.00 & 0.00 & 61 & 11 & 0 & 19 & 82 \\
\hline & & 50 & 586.68 & 1.32 & 15 & 19 & 2.66 & 3.90 & 67 & 5 & 0 & 7 & 11 \\
\hline & & 75 & 600.00 & 1.99 & 16 & 20 & 3.63 & 5.06 & 79 & 2 & 0 & 1 & 0 \\
\hline \multirow{9}{*}{ Ger } & 100 & 25 & 21.52 & 0.00 & 6 & 5 & 0.00 & 0.00 & 43 & 4 & 0 & 143 & 256 \\
\hline & & 50 & 45.97 & 0.00 & 6 & 5 & 0.01 & 0.01 & 67 & 4 & 0 & 169 & 252 \\
\hline & & 75 & 70.71 & 0.00 & 6 & 6 & 1.51 & 1.00 & 98 & 4 & 1 & 171 & 249 \\
\hline & 250 & 25 & 347.82 & 0.36 & 13 & 14 & 0.00 & 0.00 & 274 & 9 & 1 & 243 & 134 \\
\hline & & 50 & 407.43 & 0.37 & 12 & 13 & 2.94 & 4.15 & 172 & 6 & 1 & 165 & 107 \\
\hline & & 75 & 449.38 & 0.55 & 12 & 13 & 3.03 & 4.25 & 158 & 6 & 0 & 98 & 92 \\
\hline & 500 & 25 & 431.51 & 0.52 & 17 & 20 & 0.00 & 0.00 & 59 & 9 & 0 & 31 & 96 \\
\hline & & 50 & 542.32 & 0.58 & 17 & 18 & 1.34 & 2.78 & 65 & 3 & 0 & 13 & 43 \\
\hline & & 75 & 600.00 & 2.50 & 23 & 28 & 8.13 & 6.27 & 79 & 1 & 0 & 1 & 0 \\
\hline \multirow{9}{*}{ ND-I } & 100 & 25 & 37.22 & 0.00 & 7 & 10 & 0.00 & 0.00 & 94 & 5 & 0 & 282 & 256 \\
\hline & & 50 & 31.60 & 0.00 & 6 & 10 & 6.44 & 11.85 & 66 & 3 & 0 & 99 & 256 \\
\hline & & 75 & 48.10 & 0.00 & 6 & 10 & 6.45 & 11.82 & 91 & 3 & 0 & 100 & 256 \\
\hline & 250 & 25 & 296.20 & 0.07 & 16 & 18 & 0.00 & 0.00 & 103 & 5 & 0 & 287 & 153 \\
\hline & & 50 & 384.78 & 0.12 & 15 & 18 & 7.32 & 8.22 & 103 & 5 & 1 & 167 & 115 \\
\hline & & 75 & 330.24 & 0.13 & 14 & 16 & 8.86 & 10.71 & 106 & 4 & 1 & 106 & 151 \\
\hline & 500 & 25 & 543.29 & 1.98 & 25 & 38 & 0.00 & 0.00 & 77 & 15 & 0 & 30 & 33 \\
\hline & & 50 & 584.45 & 2.01 & 24 & 33 & 0.67 & 5.07 & 63 & 7 & 0 & 6 & 12 \\
\hline & & 75 & 600.00 & 2.38 & 23 & 36 & 12.25 & 18.06 & 79 & 2 & 0 & 1 & 0 \\
\hline
\end{tabular}

Table 2: Statistics of solution characteristics and algorithmic performance for different values of $n$ and $|K|$ (Groups US, Germany and ND-I)

the average value of $\Delta \omega \%$, can be explained by the fact that not all instances are solved to optimality (especially for $n=500$ and $|K|=75$ ), so the non-optimal first-stage solutions are such that the corresponding recovery costs are sub-optimally high.

\subsection{Trans Instances: Algorithmic Performance}

Assessment of Algorithmic Enhancements In $\$ 3$ we have described several enhancements for our algorithm: cut strengthening based on dual-lifting, scenario sorting, zero-half cuts, matheuristic generation of cuts and branching priorities on auxiliary variables. In Figure 7 we show box-plots of the gaps attained when solving instances of group US with $n=250$ when incrementally including the proposed techniques. Each box represents the 


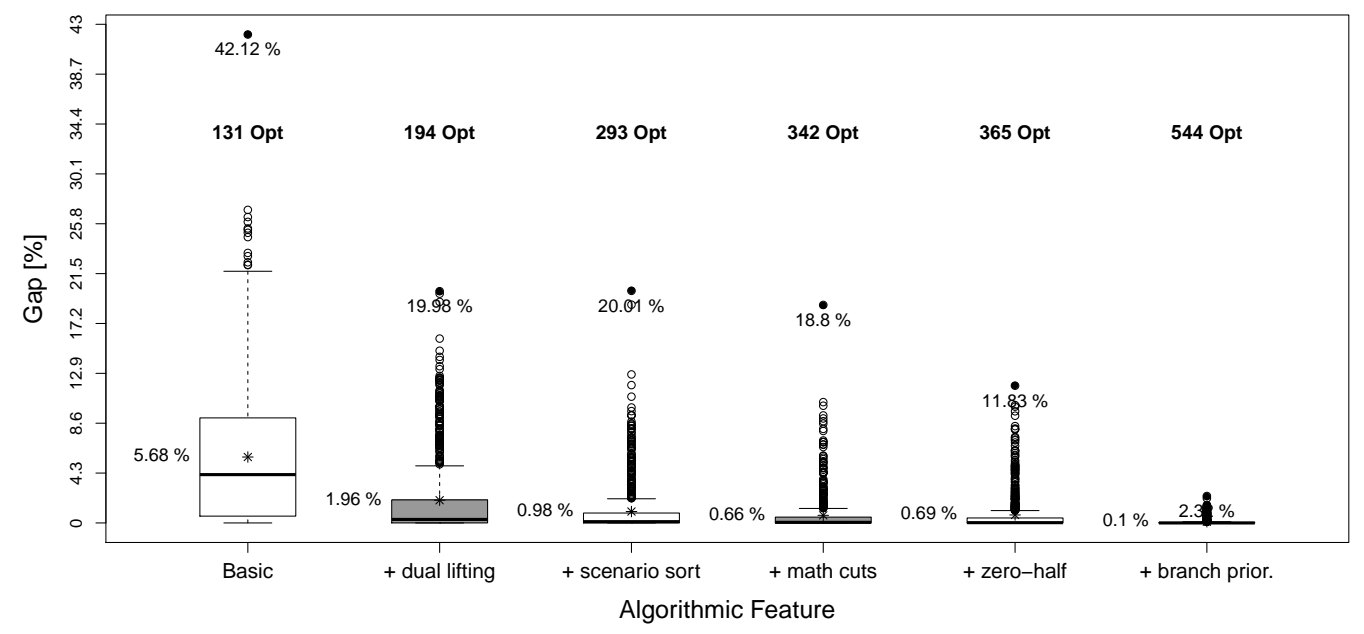

Figure 7: Influence of the special enhancement strategies on the algorithmic performance (Group US, $n=250$ )

distribution of the obtained gaps over a set of 678 instances. The first box-plot corresponds to the basic setting of the algorithm, that is, with the cuts of type (LS) and (i-LS); the second box-plot shows the gaps obtained when using the strengthening technique based on dual variables (i.e., when adding $(l-\mathrm{LS})$ instead of $(\overline{\mathrm{LS}})$ ); in the third box-plot we display the gaps obtained when adding the strategy of scenario sorting; the fourth box-plot shows the gaps attained when adding cuts generated by our matheuristic approach; the gaps attained when strengthening found cuts using zero-half cuts are given in the fifth box-plot; finally, in the sixth box-plot we show the gaps obtained when imposing higher branching priorities on the auxiliary variables (this last configuration is our default one). The bold points are the maximum gaps, asterisks are the average gaps and on top of each box we show the total number of instances (out of 678) that were solved to optimality.

The results clearly demonstrate that all the proposed techniques contribute to the effectiveness of the algorithm and complement each other: the average gap decreases, more instances are solved to optimality and the performance is more stable. In terms of the marginal contribution to the algorithmic performance, the strengthening technique based on dual-lifting and imposing higher branching priorities on the auxiliary variables seem to be the techniques that produce largest improvements of the algorithmic performance. Using the basic strategy, only 131 instances can be solved to optimality. On the contrary, using a combination of our enhancement methods, 544 instances are solved to optimality within the same time limit.

More detailed indicators of the effectiveness of the considered cuts and their algorithmic performance are provided in Table 2 . In columns $\#(l$-LS $)$, $\#(l \text {-LS })_{\mathrm{MH}}$ and $\#(i$-LS $)$ we report the average number of $L$-shaped Cuts, $L$-shaped Cuts found via the matheuristic approach, and integer $L$-shaped Cuts, respectively, that are added during the optimization process. Column \#BBN reports the average number of enumeration nodes explored within the running time.

It is remarkable that (cf. column \#( $i$-LS $)$ ), integer $L$-shaped cuts are added in very rare cases. In a more detailed analysis we observed that whenever the current solution $\left(\tilde{\mathbf{x}}^{0}, \tilde{\mathbf{y}}^{0}\right)$ 


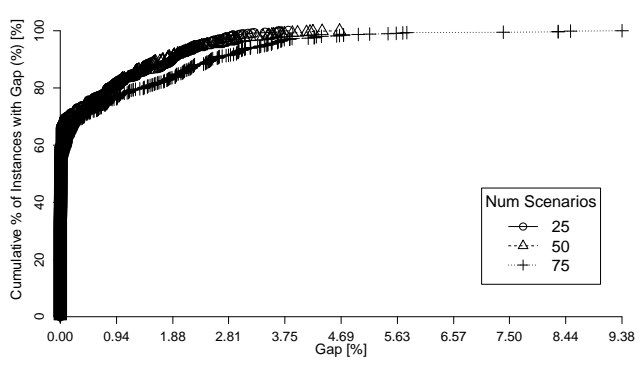

(a) Performance Profile of attained gaps

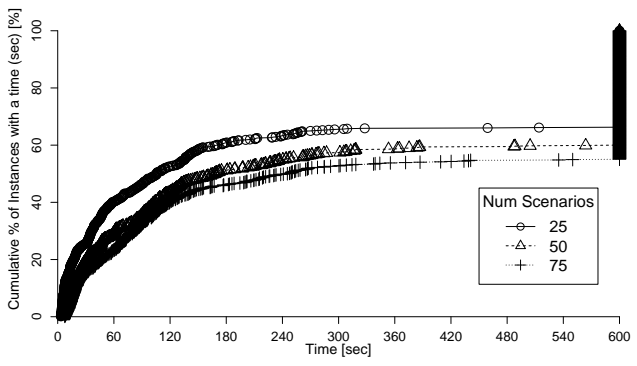

(b) Performance Profile of running times

Figure 8: Performance Profile of attained gaps and running times for different number of scenarios (Group US, 2034 instances)

was integer, usually (l-LS) were able to close the gap, so no attempt was made to find integer $L$-shaped cuts.

The number of explored enumeration nodes (column \#BBN) clearly shows that increasing the size of the instance and the number of scenarios produces a slowdown in the exploration of the search-space. This happens because more time is spent at each node solving the separation problem and performing the algorithmic enhancements described before.

The effectiveness of the proposed solution approach on the 2034 instances derived from group US is shown in Figure 8. The performance profile of the attained gaps for different values of $|K|$ in Figure 8(a) shows that (regardless of the value of $|K|$ ): (i) about $65 \%$ of the instances are solved to optimality or a very small gap is reached, (ii) for almost $80 \%$ of the instances a gap of less than $1.5 \%$ is reached, and (iii) for almost all, expect 5 instances, the attained gap is less than 4.7\%. As for the running times, Figure 8(b) shows that: (i) between $20 \%$ and $40 \%$ of the instances can be solved in less than 60 seconds, (ii) about $50 \%$ can be solved in less than 300 seconds, and (iii) for almost $45 \%$ of the instances the time limit is reached. Detailed performance profiles of the attained gaps for different values of $n$ are provided in the Appendix (Figure 10). The observed behavior is not very different in the case of the group Germany (Figure 11 in the Appendix), nor in the case of the group ND-I (Figure 12 in the Appendix).

Recall that for our branch-and-cut approach we have disabled some CPLEX features (pre-processing, heuristics and general-purpose cutting planes) in order to get a better assessment of the proposed techniques. For the sake of completeness, we have performed some experiments where all CPLEX parameters are set to their default values. In Table 6 in the Appendix we report statistics on the algorithmic performance when solving instances with $n=100$ of groups US, Germany and ND-I with the default CPLEX settings. Comparing this table with Table 2, one observes that enabling these CPLEX features does not produce any improvement on the algorithmic performance; moreover, it actually deteriorates it: fewer instances are solved to optimality within the same time limit and the attained gaps are slightly worse.

As mentioned above, formulation (1)-(2) can also be solved directly through any stateof-the-art MIP solver such as CPLEX. Nonetheless, this straightforward strategy cannot be 


\begin{tabular}{|c|c|c|c|c|c|c|c|c|}
\hline \multirow[b]{2}{*}{ Group } & \multirow[b]{2}{*}{$k$} & \multicolumn{2}{|c|}{ Opt. Times } & \multicolumn{3}{|c|}{ Attained Gaps } & \multicolumn{2}{|c|}{ B\&C Indicators } \\
\hline & & Ave. & $\# \mathrm{Opt}$ & Ave. & $\max$ & \#Nopt & $\# \mathrm{BBN}$ & \#CPX Cuts \\
\hline \multirow{3}{*}{ US } & 25 & 17.43 & 256 & - & - & 0 & 213 & 14 \\
\hline & 50 & 36.08 & 237 & 39.56 & 84.34 & 19 & 182 & 26 \\
\hline & 75 & 54.16 & 221 & 62.72 & 99.1 & 35 & 190 & 22 \\
\hline \multirow{3}{*}{ Ger } & 25 & 14.20 & 256 & - & - & 0 & 72 & 9 \\
\hline & 50 & 41.87 & 244 & 17.8 & 42.85 & 12 & 133 & 19 \\
\hline & 75 & 66.25 & 206 & 31.6 & 98.01 & 50 & 269 & 25 \\
\hline \multirow{3}{*}{$N D-I$} & 25 & 7.72 & 253 & - & - & 0 & 138 & 13 \\
\hline & 50 & 38.10 & 256 & - & - & 0 & 131 & 17 \\
\hline & 75 & 54.40 & 228 & 49.48 & 99.63 & 28 & 175 & 31 \\
\hline
\end{tabular}

Table 3: Algorithmic performance of CPLEX when solving the compact model. Trans Instances with $n=100$ (256 instances per row).

applied successfully, even to our smallest instances $(n=100)$. In Table 3 (cf. Table 7 ) we report statistics on the performance of CPLEX with default configuration when solving instances of class Trans with $n=100$ (within the same time limit of 600 seconds). We observe that much less instances are solved to optimality, and the gaps of the unsolved instances can be as high as $99 \%(!)$ and average gaps can range from $17.0 \%$ to more than $60.0 \%$. In the table we also report the number of explored enumeration nodes (\#BBN) and the number of cuts added by the solver (\#CPX Cuts). What seems surprising is the small number of general-purpose cuts added during the optimization with respect to the number of explored nodes; this means that cutting planes as those included in CPLEX are insufficient to tackle the structure of the RRUFL (at least for the considered instances) and the lower-bound improvement mainly relies on branching.

\subsection{Dis Instances: Solutions and Algorithmic Performance}

Solutions Dis class is intended to represent situations of natural disasters in which different number of cities are likely to need assistance $(t=\{0.25,0.50,0.75\})$, few cities are in conditions to host a facility, a portion of the allocation links can be heavily damaged $(f=\{0.00,0.10,0.25,0.50\})$ and the attractiveness of a location depends more on its position than on its economical characteristics.

As in the case of Trans instances, the structure of the first-stage solutions strongly depends on the instance definition. Figure 9 displays solutions of instances of group Philippines considering different combinations of $(t, f)$. We can observe that for a fixed value of $t$ (Figures 9(a) $9(\mathrm{c})$ for $t=0.50$ and Figures $9(\mathrm{~d})$ 9(f) for $t=0.75)$, a larger first-stage component is defined when increasing $f$, i.e., more facilities are opened and more allocations are defined. This behavior is expected due to the dramatic effect produced by the presence of road failures; it is better to define robust first-stage allocations to prevent from very high transportation times in the second stage.

Note that, from a practical point of view, if a given city $i$ is assigned in the first stage to a facility $j$, the actual allocation cost (the one incurred when assistance comes from $j$ to $i$ after the disaster) will still be scenario dependent (chosen roads might be damaged in any case). However, this first-stage decision can help to decision makers (i) to define preventive plans 


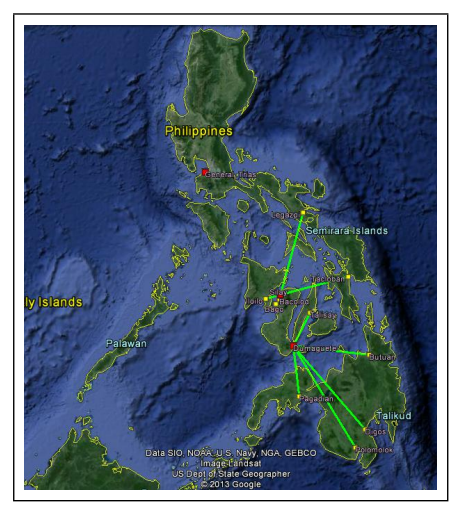

(a) $(0.50,0.10),\left|\mathbf{y}^{0}\right|=3,\left|\mathbf{x}^{0}\right|=11$

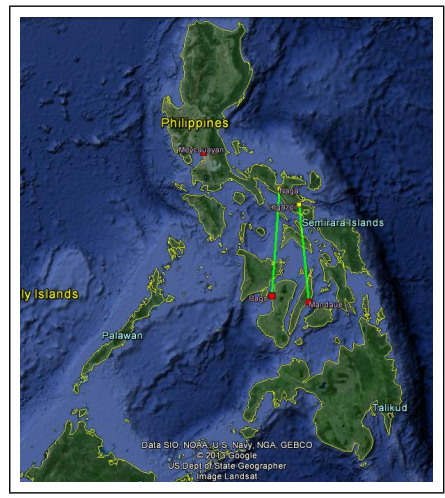

(d) $(0.75,0.10),\left|\mathbf{y}^{0}\right|=3,\left|\mathbf{x}^{0}\right|=2$

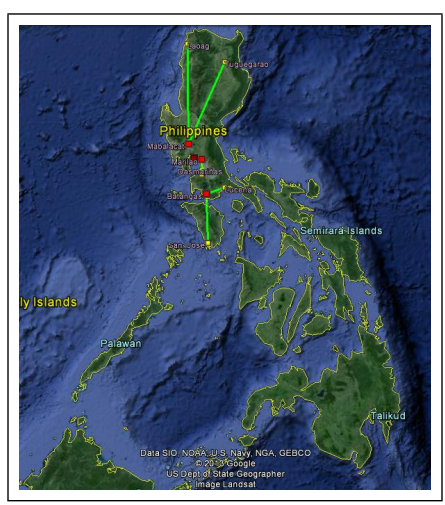

(b) $(0.50,0.25),\left|\mathbf{y}^{0}\right|=4,\left|\mathbf{x}^{0}\right|=5$

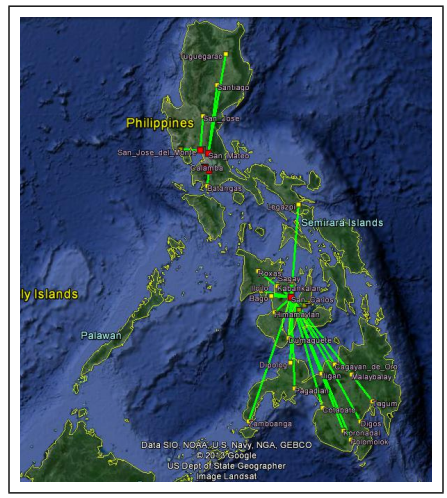

(e) $(0.75,0.25),\left|\mathbf{y}^{0}\right|=5,\left|\mathbf{x}^{0}\right|=29$

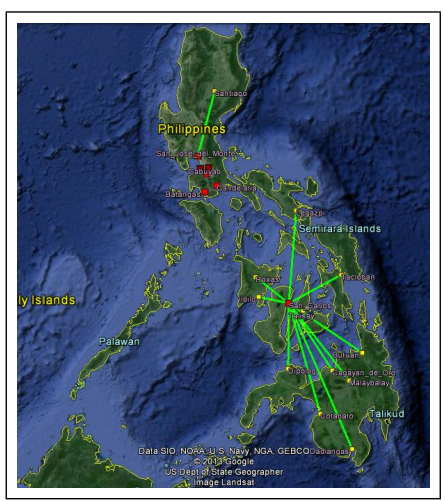

(c) $(0.50,0.50),\left|\mathbf{y}^{0}\right|=7,\left|\mathbf{x}^{0}\right|=13$

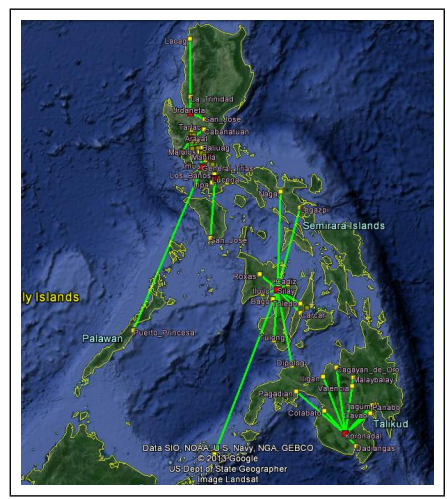

(f) $(0.75,0.50),\left|\mathbf{y}^{0}\right|=7,\left|\mathbf{x}^{0}\right|=58$

Figure 9: Solutions considering different combinations of $(t, f)$ (Group Philippines, $\sigma_{3}, \sigma_{4}=1,|K|=50$ )

to endure some roads, (ii) to have in mind how to access the affected areas regardless of the presence of failures, and (iii) to make sure that if a given allocation should be re-defined, this re-allocation will be economically efficient (due to the worst-case emphasis of the model).

Figures $9(\mathrm{a}) 9(\mathrm{f})$ have been produced by transforming our solutions into kml files that can be displayed with the Google Earth free software [see 27].

In Table 4 (equivalent to Table 2) additional information on solutions' structure is provided. As in the case of Trans instances, we can see that the number of scenarios does not change the average values of $\left|\mathbf{y}^{0}\right|$ and $\left|\mathbf{x}^{0}\right|$, which again shows that our model tackles uncertainty in a way that cost structure influences more the characteristics of first-stage solutions than the uncertainty. The values reported in columns $\triangle O P T \%$ and $\Delta \omega \%$ reinforce the previous observation: There is an important increment of the total cost of the solutions $(\triangle O P T \%$ ) when increasing $|K|$ but most of this increment is due to the second-stage component $(\Delta \omega \%)$. The marginal difference between $\Delta \omega \%$ and $\triangle O P T \%$ is due to the robustness cost of the corresponding first-stage solutions. Note that the values of $\triangle O P T \%$ and $\Delta \omega \%$ are one order of magnitude higher than those obtained for Trans; this can be explained by the nature of the Dis instances, where interdiction of transportation links induces higher allocation/re-allocation costs in the second stage.

Further insights on the influence of the cost structure on the first-stage solutions are shown in the Appendix: Figures 13 and 14 (Bangladesh group), Figures 16 and 17 (Philippines 


\begin{tabular}{|c|c|c|c|c|c|c|c|c|c|c|c|c|c|}
\hline Type & $n$ & $|K|$ & Time $[\mathrm{s}]$ & $\operatorname{Gap}(\%)$ & $\left|\mathbf{y}^{0}\right|$ & $\left|\mathbf{x}^{0}\right|$ & $\triangle O P T \%$ & $\Delta \omega \%$ & $\#(l-\mathrm{LS})$ & $\#(l-\mathrm{LS})_{\mathrm{MH}}$ & $\# i$-LS & $\# \mathrm{BBN}$ & \#Opt \\
\hline & 128 & 25 & 208.84 & 0.73 & 3 & 10 & 0.00 & 0.00 & 90 & $\overline{3}$ & 0 & 1548 & 54 \\
\hline \multirow[t]{3}{*}{ Bang } & & 50 & 308.63 & 1.81 & 3 & 11 & 23.50 & 23.60 & 138 & 3 & 0 & 1128 & 42 \\
\hline & & 75 & 293.61 & 1.75 & 3 & 10 & 29.69 & 30.82 & 145 & 3 & 0 & 665 & 43 \\
\hline & 100 & 25 & 169.79 & 0.31 & 3 & 12 & 0.00 & 0.00 & 120 & 3 & 0 & 2126 & 61 \\
\hline \multirow[t]{3}{*}{ Phi } & & 50 & 265.63 & 1.53 & 3 & 12 & 29.30 & 32.15 & 119 & 2 & 0 & 1872 & 52 \\
\hline & & 75 & 341.57 & 2.90 & 3 & 14 & 34.83 & 36.86 & 153 & 2 & 0 & 1480 & 39 \\
\hline & 100 & 25 & 219.90 & 0.56 & 3 & 8 & 0.00 & 0.00 & 104 & 2 & 0 & 2661 & 55 \\
\hline \multirow[t]{2}{*}{ ND } & & 50 & 249.05 & 1.74 & 3 & 7 & 10.39 & 13.05 & 133 & 2 & 0 & 1394 & 47 \\
\hline & & 75 & 294.59 & 2.75 & 3 & 7 & 22.64 & 25.12 & 142 & 2 & 0 & 1105 & 39 \\
\hline
\end{tabular}

Table 4: Statistics of solution characteristics and algorithmic performance for different values of $|K|$ (Groups Bangladesh, Philippines and ND-II)

\begin{tabular}{|c|c|c|c|c|c|c|c|c|c|c|c|c|c|c|}
\hline \multirow[b]{3}{*}{$|K|$} & \multicolumn{4}{|c|}{ Bangladesh-128 } & \multicolumn{5}{|c|}{ Philippines-100 } & \multicolumn{5}{|c|}{ ND-II-100 } \\
\hline & Opt. & Times & Attained & Gaps & Opt. & Times & Att & ained & Gaps & Opt. & Times & Att & ained & Gaps \\
\hline & Ave. & \#Opt & Ave. max & \#Nopt & Ave. & \#Opt & Ave. & $\max$ & \#Nopt & Ave. & \#Opt & Ave. & $\max$ & \#Nopt \\
\hline 25 & 78.45 & 54 & 2.927 .43 & 18 & 92.21 & 61 & 2.04 & 3.93 & 11 & $\overline{102.41}$ & 55 & 2.38 & 5.09 & 17 \\
\hline 50 & 100.51 & 42 & 4.339 .75 & 30 & 137.03 & 52 & 5.48 & 15.11 & 20 & 62.38 & 47 & 5.00 & 9.20 & 25 \\
\hline 75 & 86.98 & 43 & 4.339 .94 & 29 & 122.90 & 39 & 6.32 & 15.60 & 33 & 36.16 & 39 & 6.00 & 10.87 & 33 \\
\hline
\end{tabular}

Table 5: Running times needed for optimality and attained gaps when reaching the time limit for different values of $|K|$ (Groups Bangladesh, Philippines and ND-II)

group), and Figures 19 and 20 (ND-II group). From these figures we can see that the average values of $\left|\mathbf{y}^{0}\right|$ and $\left|\mathbf{x}^{0}\right|$ depend more on factors $t$ and $f$ (as previously shown in the examples) than on $\left(\sigma_{3}, \sigma_{4}\right)$ (the second-stage set-up and penalty factors).

Algorithmic Performance As in the case of Trans instances, one can identify the Effort for Robustness when solving Dis instances. From columns Time [s], Gap (\%) and \#Opt in Table 4 we observe that the greater the value of $|K|$ : (i) the larger the average running time, (ii) the greater the average attained gap, and (iii) the fewer instances are solved to optimality. From columns \#(l-LS) and $\# B B N$, we observe that, compared with Trans instances of almost the same size, much more $l$-LS $)$ cuts are added but also much more nodes are explored. This means that, on average, fewer cuts are added per enumeration node. This can be explained by the increase of numerical instability due to the presence of coefficients with different orders of magnitude. These differences lead to weaker or non-violated cuts. Therefore, our scenario sorting strategy interrupts the cut-generation cycle and forces more branching. A similar argument applies for explaining the small amount of heuristically generated cuts (column \#(l-LS$\left.)_{\mathrm{MH}}\right)$ and of integer $L$-shaped cuts (column \#(i-LS)$)$.

Table 5 reports more details regarding the algorithmic performance. The results indicate that Dis instances are more difficult to solve than Trans instances. Even if the running times for reaching optimality are still quite reasonable, the attained gaps are high (especially when considering the maximum values). The additional difficulty of these instances is explained by their more complex structure entailed by the presence of link failures (that can be very different from one scenario to another). 
The relatively high average gaps, according to Table 5, are a consequence of the presence of a few outliers with high gaps. The performance profiles of the gaps attained for different $|K|$ are shown in the Appendix in Figures 15, 18, and 21 corresponding to groups Bangladesh, Philippines, and ND-II respectively. One can conclude that in all cases the following pattern is observed: (i) for at least $60 \%$ of the instances optimality or a very small gap is reached (regardless of the value of $|K|$ ); (ii) for $75-85 \%$ of the instances a gap below $5 \%$ is attained (regardless of the value of $|K|$ ); (iii) for at most $5 \%$ of the instances gaps above $10 \%$ are obtained (only for $|K|=\{50,75\}$ ).

The previously described instability of the attained gaps and their dependence on the instance structure is clearly depicted in the complementary charts provided in the Appendix. One observes that factors $t$ and $f$ (Figures 13, 16 and 19) have more influence on the stability of the algorithmic performance, than factors $\sigma_{3}$ and $\sigma_{4}$ (Figures 14, 17 and 20). These results respond to two facts: (i) parameter $t$ plays an important role in the very structure of the instance (it defines the size of $R$ ), and (ii) parameter $f$ produces a similar effect since it defines the number of connecting links that become almost unavailable (their transportation times become 100 times larger).

\section{Conclusions}

The UFL is a classical combinatorial optimization problem of an enormous practical and theoretical relevance. Its simplicity and versatility makes it suitable to model different problems of real-world decision making. Nonetheless, when truly implementable solutions are sought, the consideration of uncertainty is unavoidable. For the UFL under different sources of uncertainty, we applied a new recoverable robust optimization approach (RRO) that falls within the framework of $2 \mathrm{SRO}$. In this new concept, a robust solution is sought such that it can be recovered (i.e., rendered feasible using a limited set of recovery actions) once the uncertainty is revealed in a second stage. For the resulting problem, RRUFL, we designed a branch-and-cut framework based on Benders decomposition and we included several tailored enhancements to improve its performance.

The proposed algorithm was extensively tested on more than 7500 realistic instances divided into two groups. The results show the efficacy of the algorithm in finding good quality solutions within a short running time. Moreover, the results demonstrate the strong influence of the instance cost structure on both the algorithmic performance and solution characteristics. Our computational study also illustrates how robustness and recoverability are expressed in the structure of optimal solutions, and it demonstrates the benefits of RRO when compared to a RO model without recovery.

Finally, the obtained results indicate that solving the RRUFL is a not an easy task for general purpose MIP solvers. To cope with the size of realistic instances, it is inevitable to use more sophisticated decomposition techniques, like the one presented in this study.

[1] D. Adjiashvili. Structural Robustness in Combinatorial Optimization. PhD thesis, ETH ZURICH, 2012.

[2] M. Albareda-Sambola, E. Fernández, and F. Saldanha-da Gama. The Facility Location problem with Bernoulli demands. Omega, 39(3):335-345, 2011. 
[3] M. Albareda-Sambola, A. Alonso-Ayuso, L. Escudero, E. Fernández, and C. Pizarro. Fix-and-relax-coordination for a multi-period location-allocation problem under uncertainty. Computers \& OR, 40(12):2878-2892, 2013.

[4] S. Alumur, S. Nickel, and F. Saldanha-da Gama. Hub location under uncertainty. Transportation Research Part B: Methodological, 46(4):529-543, 2012.

[5] E. Álvarez-Miranda, I. Ljubić, S. Raghavan, and P. Toth. The recoverable robust twolevel network design problem. Submitted to INFORMS J. on Computing, 2013.

[6] G. Andreello, A. Caprara, and M. Fischetti. Embedding $\{0,1 / 2\}$-cuts in a branch-andcut framework: A computational study. INFORMS Journal on Computing, 19:229-238, 2007.

[7] I. Averbakh. The minmax relative regret median problem on networks. INFORMS Journal on Computing, 17(4):451-461, 2005.

[8] A. Ben-Tal, A. Goryashko, E. Guslitzer, and A. Nemirovski. Adjustable robust solutions of uncertain linear programs. Mathematical Programming, Series A(99):351-376, 2004.

[9] A. Ben-Tal, L. El-Ghaoui, and A. Nemirovski, editors. Robust Optimization. Series in Applied Mathematics. Princeton, 1st edition, 2010.

[10] D. Bertsimas and M. Sim. Robust discrete optimization and network flows. Mathematical Programming, Series B(98):49-71, 2003.

[11] D. Bertsimas and M. Sim. The price of robustness. Operations Research, 52(1):35-53, 2004.

[12] J. Birge and F. Louveaux. Introduction to Stochastic Programming. Series in Operations Research and Financial Engineering. Springer, 2nd edition, 2011.

[13] C. Büsing. Recoverable robust shortest path problems. Networks, 59(1):181-189, 2012.

[14] C. Büsing, A. Koster, and M. Kutschka. Recoverable robust knapsacks: the discrete scenario case. Optimization Letters, 5(3):379-392, 2011.

[15] V. Cacchiani, A. Caprara, L. Galli, L. Kroon, and G. Maróti. Recoverable robustness for railway rolling stock planning. In M. Fischetti and P. Widmayer, editors, Proceedings of ATMOS 2008, volume 9 of OpenAccess Series in Informatics. Schloss Dagstuhl, 2008.

[16] A. Caprara and M. Fischetti. $\left\{0, \frac{1}{2}\right\}$-Chvátal-Gomory cuts. Mathematical Programming, 74:221-235, 1996.

[17] S. Cicerone, G. Di Stefano, M. Schachtebeck, and A. Schöbel. Multi-stage recovery robustness for optimization problems: A new concept for planning under disturbances. Information Sciences, 190:107-126, 2012. 
[18] G. Cornuéjols, G. Nemhauser, and L. Wolsey. The uncapacitated facility location problem. In P. Mirchandani and R. Francis, editors, Discrete Location Theory. WileyInterscience, 1990.

[19] T. Cui, Y. Ouyang, and Z. Shen. Reliable facility location design under the risk of disruptions. Operations Research, 58(4):998-1011, 2010.

[20] G. D'Angelo, G. Di Stefano, A. Navarra, and C. Pinotti. Recoverable robust timetables: An algorithmic approach on trees. IEEE Transactions on Computers, 60(3):433-446, 2011.

[21] M. Daskin. Network and Discrete Location: Models, Algorithms, and Applications. Wiley, 2nd edition, 2013.

[22] DDM. Department of Disaster Management of Bangladesh, Ministry of Disaster Management and Relief, Government of the Republic Bangladesh, 2014. URL http://http: //www.ddm.gov.bd/flood.php/.

[23] H. A. Eiselt and V. Marianov, editors. Foundations of Location Analysis, volume 155 of International Series in Operations Research \& Management Science. Springer, 2011.

[24] M. Fischetti and A. Lodi. Local branching. Mathematical Programming, 98(1-3):23-47, 2003.

[25] Y. Gao. Uncertain models for single facility location problems on networks. Applied Mathematical Modelling, 36(6):2592 - 2599, 2012.

[26] N. Gïlpinar, D. Pachamanova, and E. Canakoglu. Robust strategies for facility location under uncertainty. European Journal of Operational Research, 225(1):21-35, 2013.

[27] Google. Google Earth, 2014. URL http://www.google.com/intl/en/earth/.

[28] R. Hassin, R. Ravi, and F. Salman. Facility location on a network with unreliable links. In Proceedings of INOC 2009. University of Pisa, 2009.

[29] P. Kouvelis and G. Yu, editors. Robust Discrete Optimization and its Applications. Nonconvex Optimization and its Applications. Kluwer Academic Publishers, 1st edition, 1997.

[30] A. Kuehn and M. Hamburger. A heuristic program for locating warehouses. Management Science, 9(4):643-666, 1963.

[31] G. Laporte and F. Louveaux. The integer L-shaped method for stochastic integer programs with complete recourse. Operations Research Letters, 13(3):133-142, 1993.

[32] Markus Leitner, Mario Ruthmair, and Günther R. Raidl. Stabilizing branch-and-price for constrained tree problems. Networks, 61(2):150-170, 2013.

[33] A. Letchford and S. Miller. Fast bounding procedures for large instances of the simple plant location problem. Computers \&6 Operations Research, 39(5):985-990, 2012. 
[34] Q. Li, B. Zeng, and A. Savachkin. Reliable facility location design under disruptions. Computers \& OR, 40(4):901-909, 2013.

[35] C. Liebchen, M. Lübbecke, R. Möhring, and S. Stiller. Recoverable robustness. Technical Report ARRIVAL-TR-0066, ARRIVAL Project, 2007.

[36] C. Liebchen, M. Lübbecke, R. Möhring, and S. Stiller. The concept of recoverable robustness, linear programming recovery, and railway applications. In R. Ahuja, R. Möhring, and C. Zaroliagis, editors, Robust and Online Large-Scale Optimization, volume 5868 of LNCS, pages 1-/27. Springer, 2009.

[37] I. Ljubić, P. Mutzel, and B. Zey. Stochastic survivable network design problems. Electronic Notes in Discrete Mathematics, 41:245-252, 2013.

[38] T. Magnanti and R. Wong. Accelerating Benders decomposition: Algorithmic enhancement and model selection criteria. Operations Research, 29(3):464-484, 1981.

[39] PAGASA. Philippine Atmospheric, Geophysical and Astronomical Services Administration, Department of Science and Technology, Republic of the Philippines, 2014. URL http://web.pagasa.dost.gov.ph/.

[40] F. Pérez-Galarce, E. Álvarez-Miranda, A. Candia, and P. Toth. On exact solutions for the Minmax Regret spanning tree problem. Forthcoming in Computers $\&$ OR, 2014. URL http://dx.doi.org/10.1016/j.cor.2014.02.007.

[41] R. Ravi and A. Sinha. Hedging uncertainty: Approximation algorithms for stochastic optimization problems. Mathematical Programming, 108(1):97-114, 2006.

[42] W. Rei, J-F. Cordeau, M. Gendreau, and P. Soriano. Accelerating Benders decomposition by local branching. INFORMS Journal on Computing, 21(2):333-345, 2009.

[43] Z. Shen, R. Zhan, and J. Zhang. The reliable facility location problem: Formulations, heuristics, and approximation algorithms. INFORMS Journal on Computing, 23(3): 470-482, 2011.

[44] L. Snyder. Facility location under uncertainty: a review. IIE Transactions, 38(7): 547-564, 2006.

[45] L. Snyder and M. Daskin. Reliability models for facility location: The expected failure cost case. Transportation Science, 39(3):400-416, 2005.

[46] L. Snyder and M. Daskin. Stochastic p-robust location problems. IIE Transactions, 38 (11):971-985, 2006.

[47] United Nations Statistics Division. UNSD Statistical Databases, 2013. URL http: //millenniumindicators.un.org/unsd/databases.htm

[48] R. Van Slyke and R. Wets. L-shaped linear programs with applications to optimal control and stochastic programming. SIAM Journal of Applied Mathematics, 17(4): 638-663, 1967. 
[49] V. Verter. Uncapacitated and capacitated facility location problems. In H. A. Eiselt and V. Marianov, editors, Foundations of Location Analysis, volume 155 of International Series in Operations Research \& Management Science, pages 25-37. Springer, 2011.

[50] L. Zhao and B. Zeng. An exact algorithm for two-stage robust optimization with mixed integer recourse problems. Tech. Report - University of South Florida, 2012. 


\section{Appendix}

\subsection{Additional Results}

In our deafult runs of the proposed branch-and-cut approach we have disabled some CPLEX features (pre-processing, heuristics and general-purpose cutting planes) in order to get a better assessment of the proposed techniques. For the sake of completeness, we have performed some experiments where all CPLEX parameters are set to their default values. In Table 6 we report statistics on the algorithmic performance when solving instances with $n=100$ of groups US, Germany and ND-I with the default CPLEX settings.

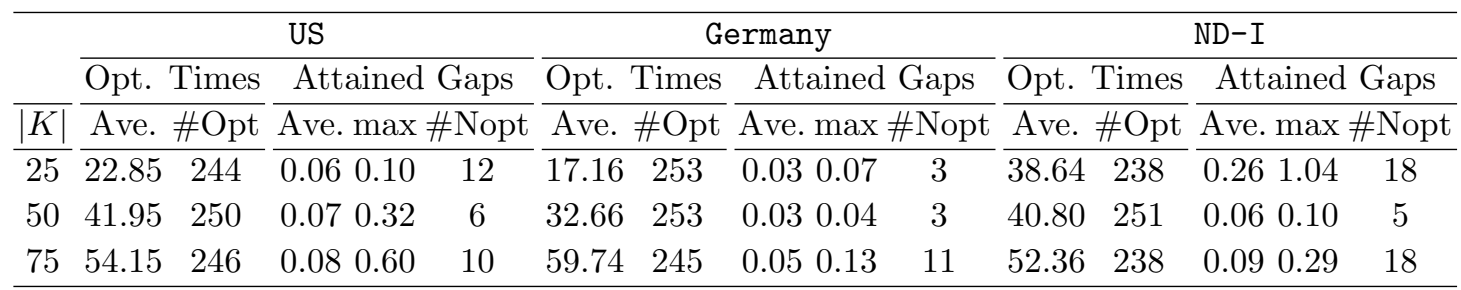

Table 6: Running times needed for optimality and attained gaps when reaching the time limit for different values of $n$ and $|K|$ when enabling CPLEX Heuristics, Cuts and Preprocessing $(n=100$, Instances US, Germany and ND-I)

\begin{tabular}{|c|c|c|c|c|c|c|c|c|c|c|c|c|c|c|c|}
\hline & \multicolumn{4}{|c|}{ US } & \multicolumn{5}{|c|}{ Germany } & \multicolumn{5}{|c|}{ ND-I } \\
\hline & & \multicolumn{2}{|c|}{ Opt. Times } & \multicolumn{2}{|c|}{ Attained Gaps } & \multirow{2}{*}{$\begin{array}{c}\text { Opt. } \\
\text { Ave. }\end{array}$} & \multirow{2}{*}{$\begin{array}{c}\text { Times } \\
\# \mathrm{Opt} \\
\end{array}$} & \multicolumn{2}{|c|}{ Attained } & \multirow{2}{*}{$\begin{array}{l}\text { Gaps } \\
\text { \#Nopt }\end{array}$} & \multicolumn{2}{|c|}{ Opt. Times } & \multicolumn{3}{|c|}{ Attained Gaps } \\
\hline$n$ & $|K|$ & Ave. & $\# \mathrm{Opt}$ & Ave. $\max$ & 4Nopt & & & Ave. & $\max$ & & Ave. & \#Opt & Ave. & $\max$ & \#Nopt \\
\hline 100 & 25 & 33.89 & 251 & 0.040 .06 & 5 & 21.52 & 256 & - & - & 0 & 37.22 & 256 & - & - & 0 \\
\hline & 50 & 63.71 & 252 & 0.590 .88 & 4 & 37.18 & 252 & 0.01 & 0.01 & 4 & 31.60 & 256 & - & - & 0 \\
\hline & 75 & 74.23 & 250 & 0.300 .95 & 6 & 55.83 & 249 & 0.02 & 0.03 & 7 & 48.10 & 256 & - & - & 0 \\
\hline$\overline{250}$ & 25 & 78.14 & 175 & 0.572 .32 & 81 & $\overline{118.23}$ & $\begin{array}{ll}3 \quad 134 \\
\end{array}$ & 0.75 & 2.54 & 122 & 91.68 & 153 & 0.16 & 1.59 & 103 \\
\hline & 50 & 118.48 & 197 & 0.252 .12 & 59 & 139.28 & $8 \quad 107$ & 0.63 & 2.17 & 149 & 120.91 & 115 & 0.22 & 1.94 & 141 \\
\hline & 75 & 132.53 & 172 & 0.221 .40 & 84 & 180.89 & 92 & 0.86 & 4.82 & 164 & 142.65 & 151 & 0.32 & 6.35 & 105 \\
\hline 500 & 25 & 156.86 & 82 & 1.623 .81 & 174 & 150.70 & 96 & 0.83 & 2.30 & 160 & 160.04 & 133 & 2.27 & 10.65 & 223 \\
\hline & 50 & 290.02 & 11 & 1.384 .66 & 245 & 256.63 & 43 & 0.70 & 2.59 & 213 & 268.23 & 312 & 2.11 & 6.19 & 244 \\
\hline & 75 & - & 0 & 1.999 .38 & 256 & - & 0 & 2.50 & 13.95 & 256 & - & 0 & 2.38 & 7.93 & 256 \\
\hline
\end{tabular}

Table 7: Running times needed for optimality and attained gaps when reaching the time limit for different values of $n$ and $|K|$ (Instances US, Germany and ND-I) 


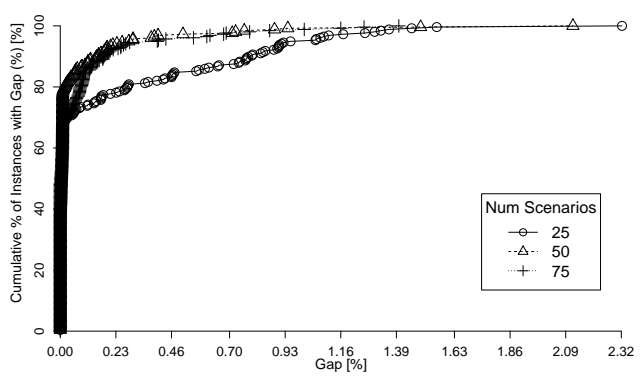

(a) US with $n=250$

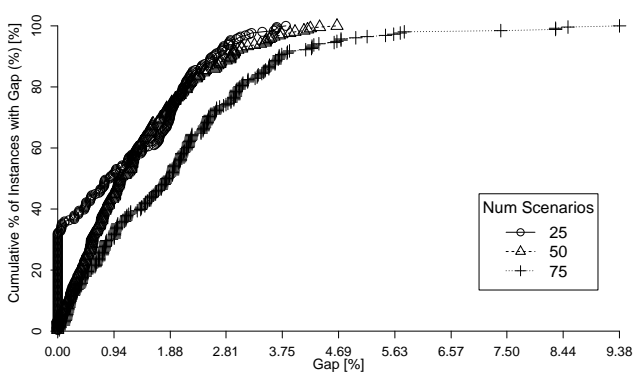

(b) US with $n=500$

Figure 10: Performance Profile of attained gaps for different $|K|$ (Group US with $n \in\{250,500\}$ )

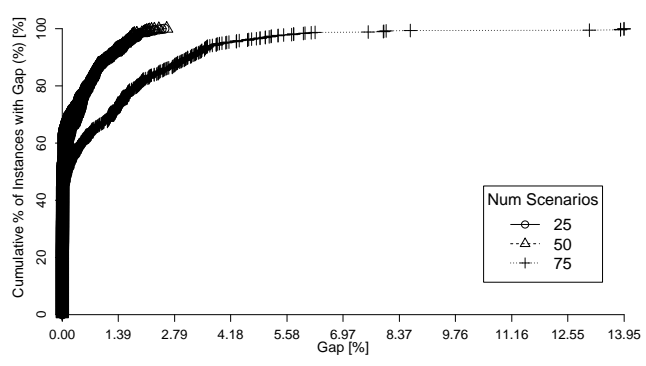

(a) Performance Profile of attained gaps

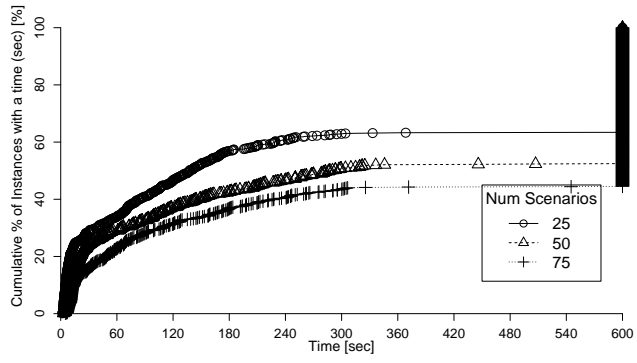

(b) Performance Profile of running times

Figure 11: Performance Profile of attained gaps and running times for different $|K|$ (Group Germany)

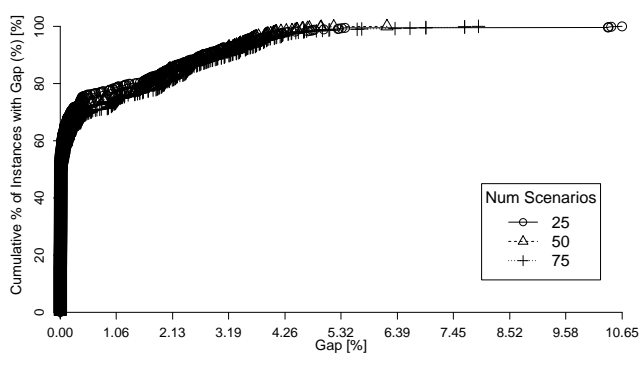

(a) Performance Profile of attained gaps

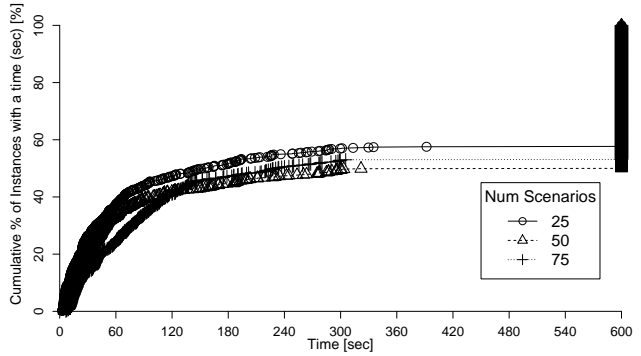

(b) Performance Profile of running times

Figure 12: Performance Profile of attained gaps and running times for different $|K|$ (Group ND-I group) 


\subsection{Detailed Results for Bangladesh Instances}

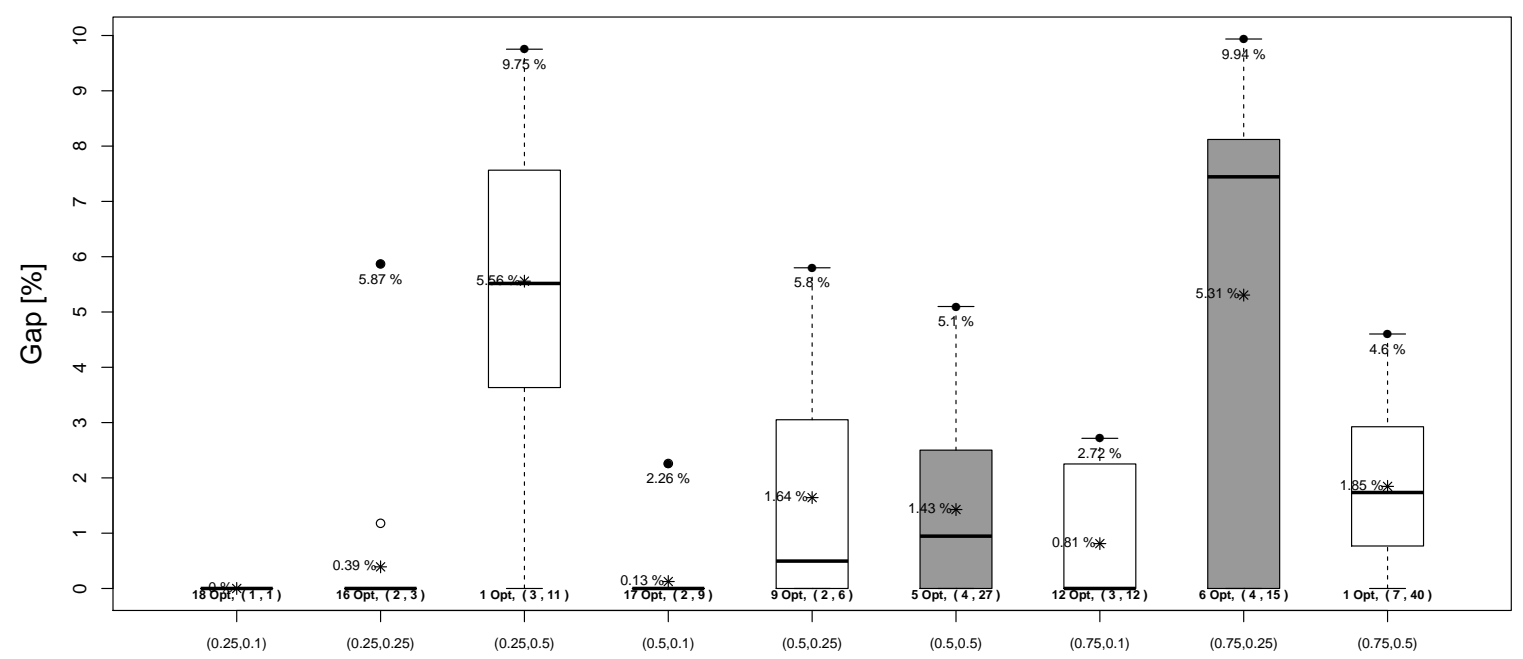

$(\mathrm{t}, \mathrm{f})$

Figure 13: Box Plot of attained gaps for different combinations of $(t, f)$ (Group Bangladesh, under each box-plot the number of optimally solved instances and the average values of $\left(\left|\mathbf{y}^{0}\right|,\left|\mathbf{x}^{0}\right|\right)$ are reported)

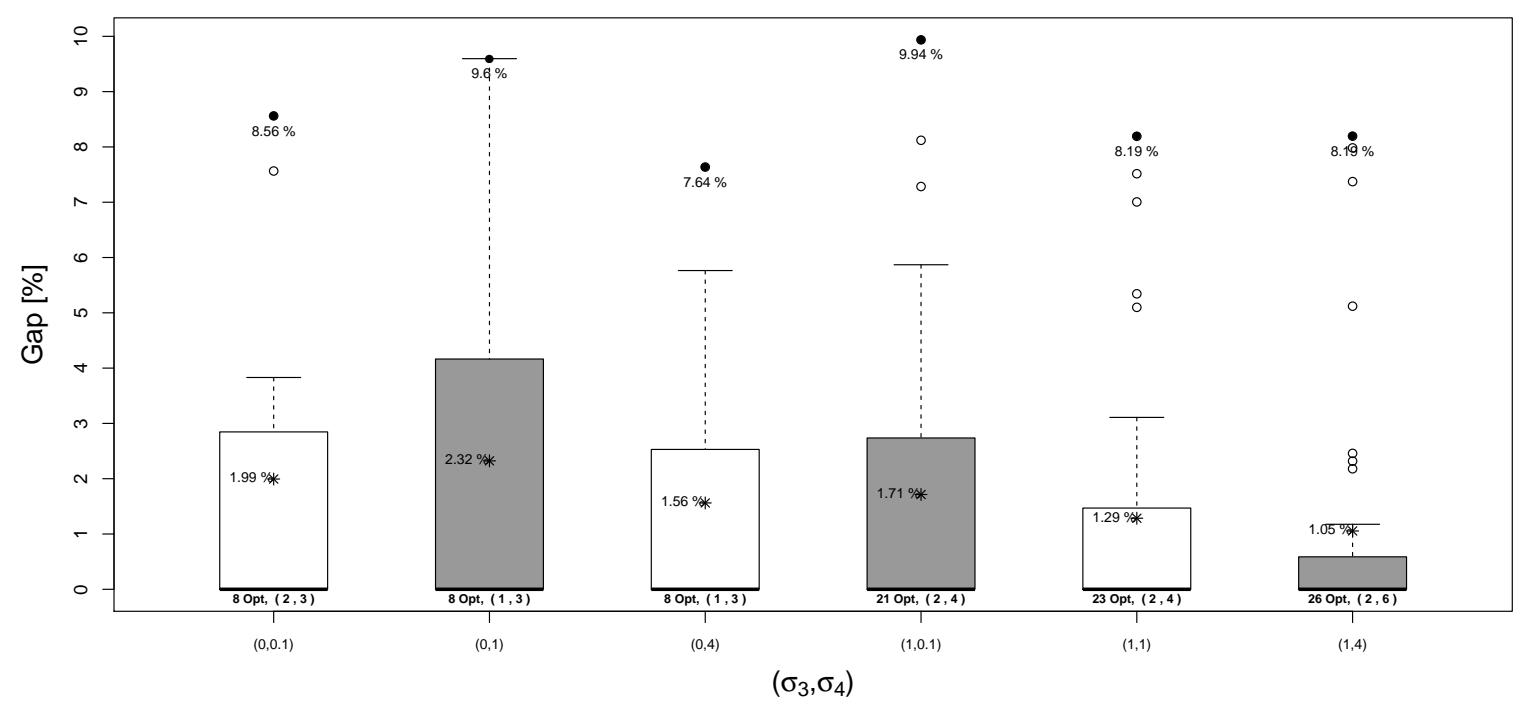

Figure 14: Box Plot of attained gaps for different combinations of $\left(\sigma_{3}, \sigma_{4}\right)$ (Group Bangladesh, under each box-plot the number of optimally solved instances and the average values of $\left(\left|\mathbf{y}^{0}\right|,\left|\mathbf{x}^{0}\right|\right)$ are reported) 


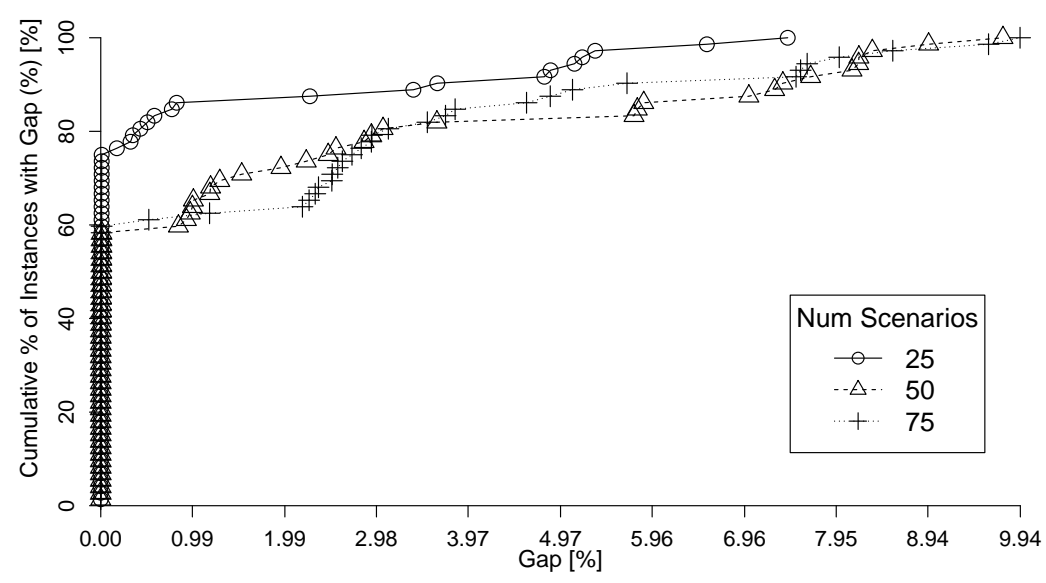

Figure 15: Performance Profile of attained gaps for different number of scenarios (Group Bangladesh) 


\subsection{Detailed Results for Philippines Instances}

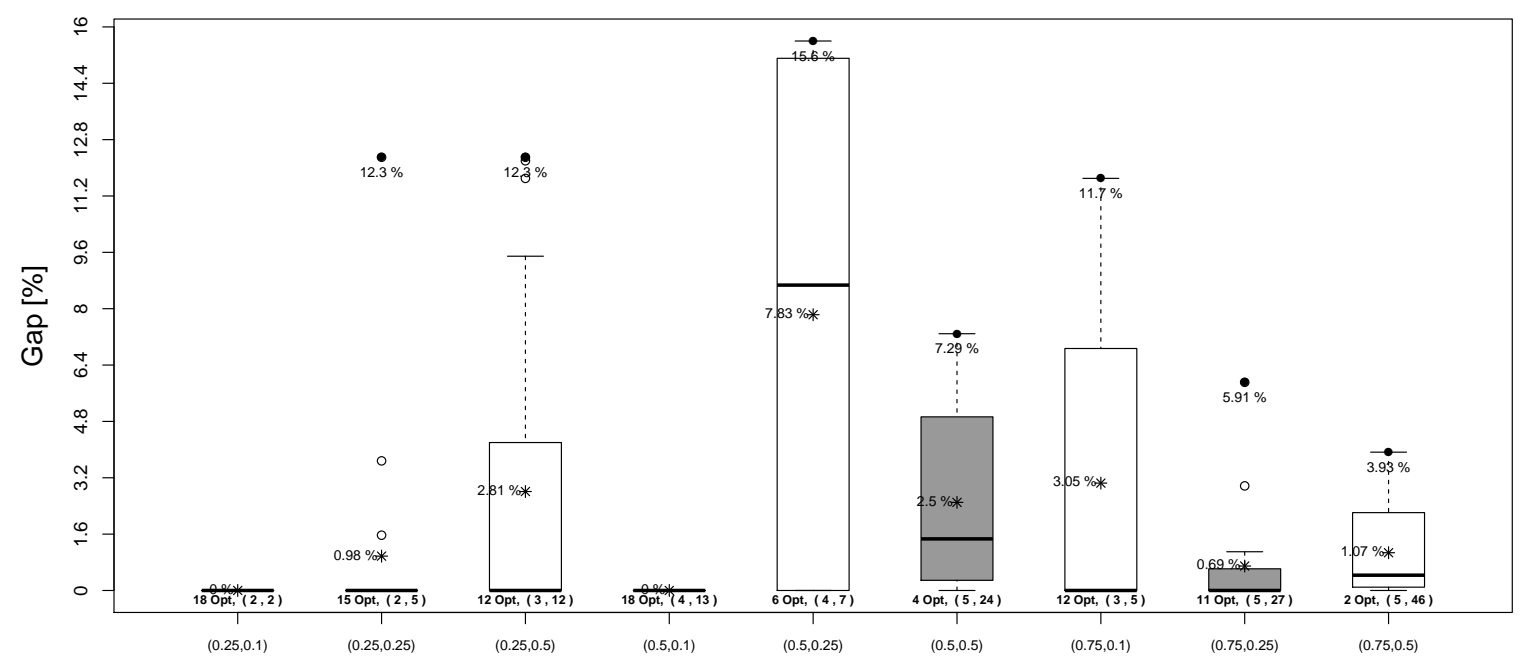

$(\mathrm{t}, \mathrm{f})$

Figure 16: Box Plot of attained gaps for different combinations of $(t, f)$ (Group Philippines, under each box-plot the number of optimally solved instances and the average values of $\left(\left|\mathbf{y}^{0}\right|,\left|\mathbf{x}^{0}\right|\right)$ are reported)

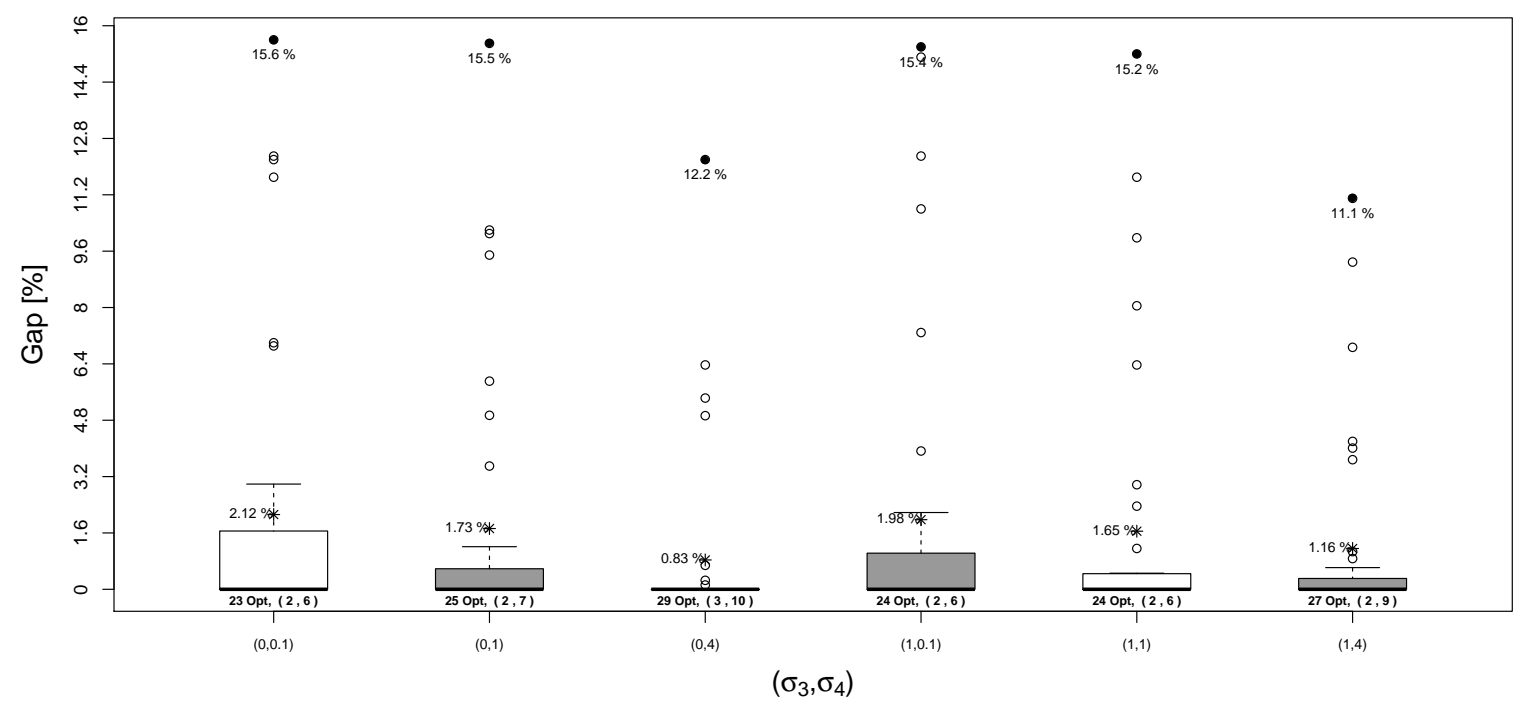

Figure 17: Box Plot of attained gaps for different combinations of $\left(\sigma_{3}, \sigma_{4}\right)$ (Group Philippines, under each box-plot the number of optimally solved instances and the average values of $\left(\left|\mathbf{y}^{0}\right|,\left|\mathbf{x}^{0}\right|\right)$ are reported) 


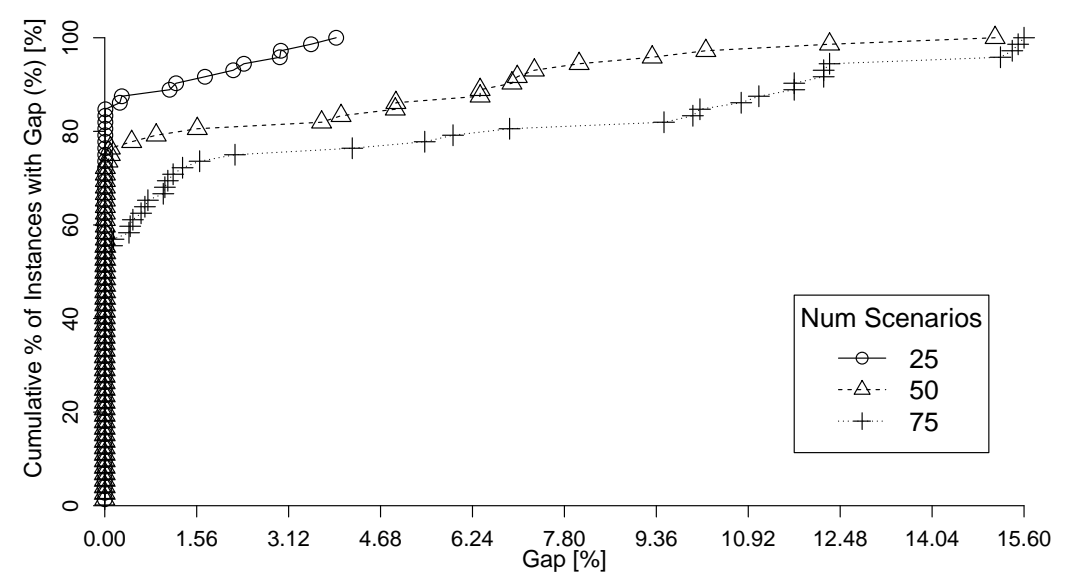

Figure 18: Performance Profile of attained gaps for different number of scenarios (Group Philippines) 


\subsection{Detailed Results for ND-II Instances}

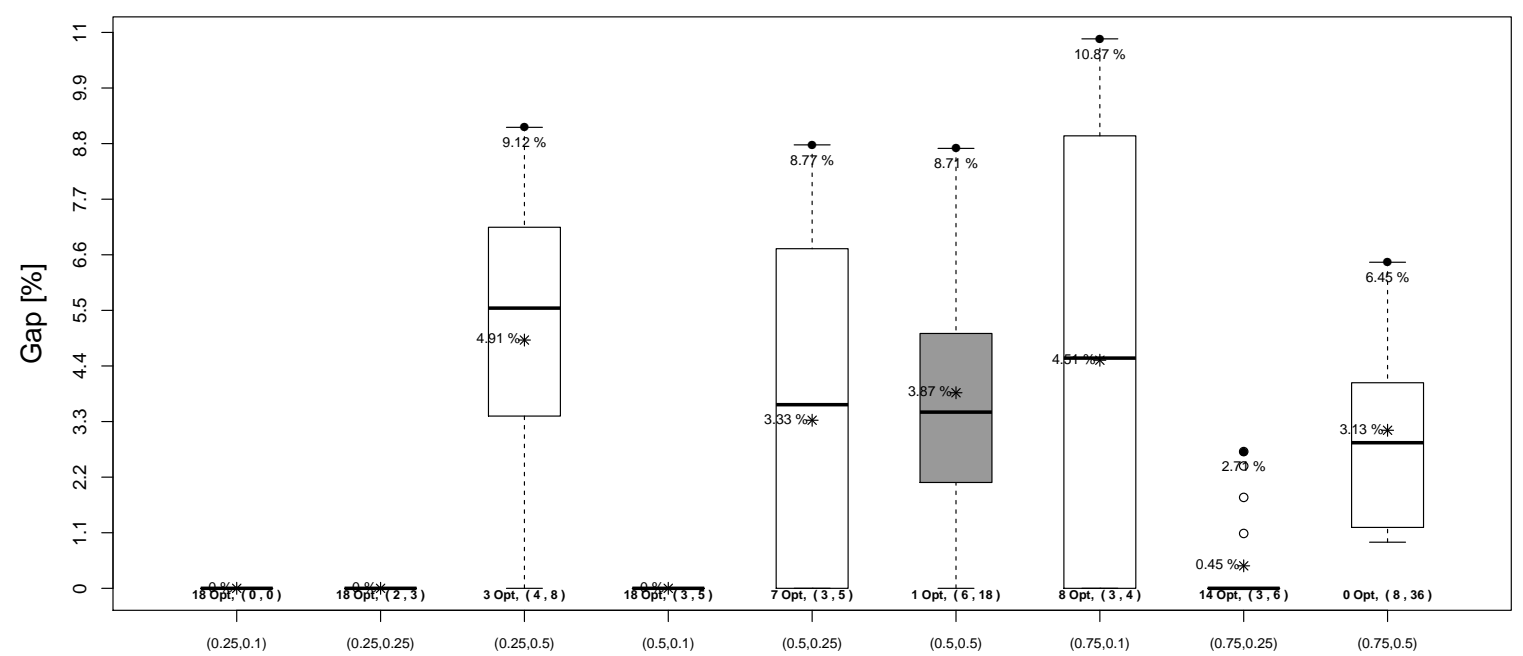

$(\mathrm{t}, \mathrm{f})$

Figure 19: Box Plot of attained gaps for different combinations of $(t, f)$ (Group ND-II, under each box-plot the number of optimally solved instances and the average values of $\left(\left|\mathbf{y}^{0}\right|,\left|\mathbf{x}^{0}\right|\right)$ are reported)

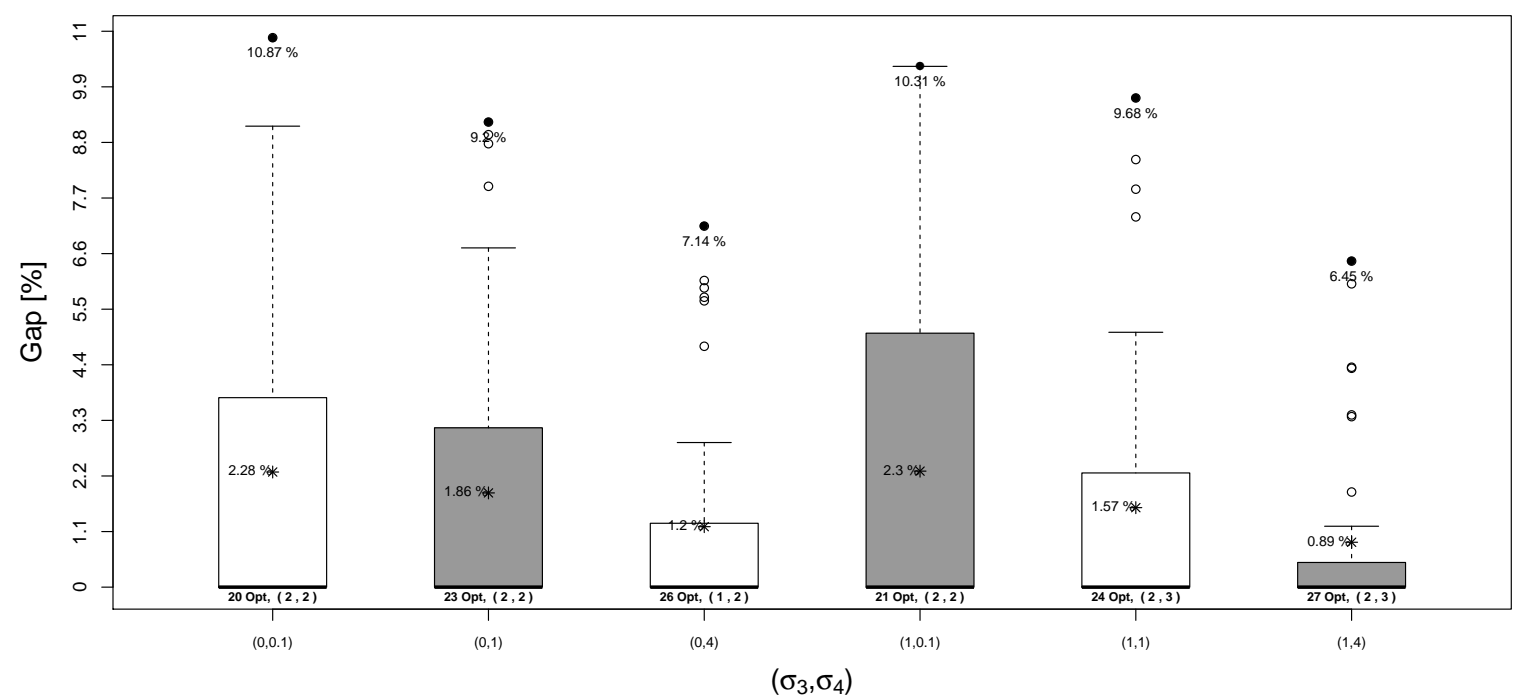

Figure 20: Box Plot of attained gaps for different combinations of $\left(\sigma_{3}, \sigma_{4}\right)$ (Group ND-II, under each box-plot the number of optimally solved instances and the average values of $\left(\left|\mathbf{y}^{0}\right|,\left|\mathbf{x}^{0}\right|\right)$ are reported) 


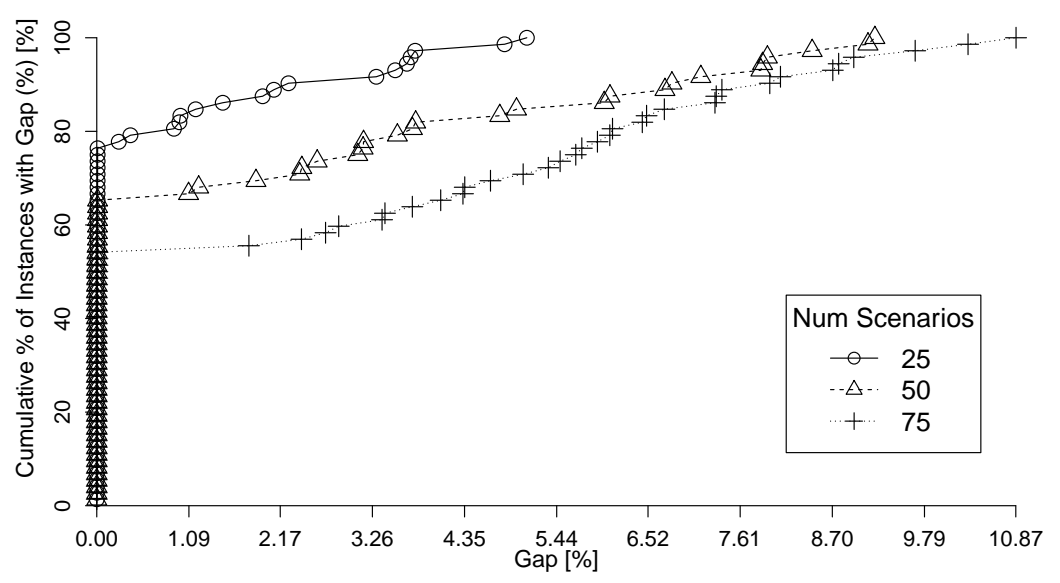

Figure 21: Performance Profile of attained gaps for different number of scenarios (Group ND-II) 